\title{
American Option Pricing: Optimal Lattice Models and Multidimensional Efficiency tests
}

\author{
Qianru Shang \\ Technological University Dublin, qianru.shang@tudublin.ie \\ Brian Byrne \\ Technological University Dublin, brian.byrne@tudublin.ie
}

Follow this and additional works at: https://arrow.tudublin.ie/buschmanart

Part of the Finance and Financial Management Commons

\section{Recommended Citation}

Shang, Q. and Byrne, B.. (2020). American Option Pricing: Optimal Lattice Models and Multidimensional Efficiency Tests (May 23, 2020). Journal of Futures Markets. Available at SSRN. doi: 10.2139/

ssrn. 3608812

This Article is brought to you for free and open access by the School of Management at ARROW@TU Dublin. It has been accepted for inclusion in Articles by an authorized administrator of ARROW@TU Dublin. For more information, please contact arrow.admin@tudublin.ie, aisling.coyne@tudublin.ie,gerard.connolly@tudublin.ie.

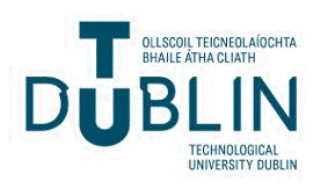




\title{
American Option Pricing: Optimal Lattice Models and Multidimensional Efficiency Tests
}

\author{
Qianru Shang ${ }^{\mathrm{a}, *}$, Brian Byrne ${ }^{\mathrm{a}}$ \\ ${ }^{a}$ Department of Accounting and Finance, College of Business, Technological University Dublin, \\ Dublin, D02 HW71, Ireland. \\ * Corresponding author. \\ Email addresses: qianru.shang@tudublin.ie (Q. Shang), brian.byrne@tudublin.ie (B. Byrne).
}

This research did not receive any specific grant from funding agencies in the public, commercial, or not-for-profit sectors. 
This paper introduces a set of lattice techniques with a view to accelerating computation time and improving the accuracy of American Option valuation. Estimation speed can be enhanced through developing a parsimonious early exercise boundary search routine combined with reliance on dynamic memory and lattice truncation. Furthermore, Black-Scholes and Richardson extrapolation modifications to the lattices can also be applied individually and/or together to improve the accuracy of lattices. In this paper, we investigate the improvement introduced by obtaining the best combination of varying features. By introducing these techniques to the Leisen-Reimer and Tian binomial model, we can achieve a level of accuracy and efficiency combined that surpass analytical analogues prominent in the literature. Significantly, the Leisen-Reimer and Tian structure can accommodate arbitrary improvements in accuracy by simply increasing the density of their own mesh. Analytical methods generally do not afford much scope for optimising speed and efficiency in a granular fashion. We also compare efficient lattice models with analytical formulae for pricing different groups of options according to the deepness of American quality and the moneyness of the options. The appropriate model is recommended for pricing particular types of the options. Lattices importantly afford an explicit tradeoff locus between accuracy and speed that can be navigated according to predetermined precision tolerance levels and option types. This should have practical relevance to trading platforms that require real-time estimates of implied volatility.

Keywords: Pricing, American Options, Lattices Methods, Optimal Exercise Boundary 


\section{Introduction}

A put (call) option provides the holder with the right to sell (buy) the underlying asset at a fixed price. If the holder can only exercise the option at the maturity, the option is referred to as being European. In contrast, an American option can be exercised early, at any time before the maturity. With the possibility of early exercise, American option pricing gives rise to an optimal exercise problem. A closed-form solution for European option pricing is available using Black and Scholes (1973). No equivalently simple closed-form solution exists for valuing American options robustly. McKean (1965) and Merton (1973) lay the foundation for the American option pricing. They showed that the American option valuation problem can be viewed as a free boundary (also known as the optimal exercise boundary) problem of a partial differential equation. Since then, varying methods have been proposed for pricing American options written on a single underlying asset, which can be largely classified into two categories: analytical approximations or numerical methods.

Approximate analytic solutions are heavily relied upon to expedite estimation. Geske and Johnson (1984) valued American options using an infinite sequence of multivariate cumulative normal terms, which was improved in Bunch and Johnson (1992) by locating two exercise points. A key limitation however relates to the inefficiency associated with adding more and more terms to capture an exact representation of the free boundary problem typical in American options. Barone-Adesi and Whaley (1987, BAW) proposed a quadratic approximation. It is known to be fast and accurate for most input values but it becomes less accurate when pricing an American option with longer maturity horizons. American options values tend to diverge more markedly from their European equivalents as maturity increases. Ju and Zhong (1999, Ju-Zhong) extended the quadratic approach and improved pricing for longer maturities instruments relative to BAW. Ju-Zhong pointed out that their approach is more accurate than the less efficient methods proposed, such as, the four-point extrapolation schemes of Geske and Johnson (1984) and Huang, et al. (1996). Li (2010) further extended the Ju-Zhong approach by suggesting an improved smoothing condition for American options. This had the effect of producing a more exact estimate of the optimal exercise price although did not guarantee a markedly better option pricing performance. The approximations of BAW, Ju-Zhong and Li models however do not in essence converge to the true value. In addition to quadratic approximation techniques, Bjerksund and Stensland (1993, Bjerksund93) obtained an approximation by simplifying the optimal exercise strategy with a unique flat early exercise boundary. Bjerksund and Stensland (2002, Bjerksund02) made further improvement by incorporating the second bound. These approaches represent accurate and efficient approximations to the American option value. Zhu (2006) proposed an exact solution by making use of a Taylor series expansion potentially leading to an infinite many terms. Zhu's approach points to the existence of a closed-form solution for American option pricing but is hard to implement in terms of computational costs (Kim et al. 2013; Chen and Joshi 2012; Medvedev and Scaillet 2010). In this paper, we compare our efficient lattice models with analytical approximations prominent in the literature and 
we show that our most refined models surpass the accuracy and speed of these commonly used analytical frameworks.

Numerical techniques are regularly used as benchmarks for analytical approximations on account of the high level of estimation accuracy. Brennan and Schwartz (1977) and Dempster and Hutton (1999) transformed the partial differential equation into linear equations and linear programming problems respectively using finite difference methods. Wu and Kwok (1997) and Nielsen et al. (2002) converted free-boundary problems into nonlinear problems using front-fixing methods. Lattice models are generally more relied upon relative to other numerical techniques used for the valuation of American options. In their seminal paper, Cox, Ross, Rubinstein (1979, CRR) proposed a binomial model and noted that higher accuracy could be achieved by incorporating a larger number of time steps. Broadie and Detemple (1996) obtained the "true" value of American options by elaborating a 15,000-step benchmark binomial model for CRR. Using probabilistic methods, Amin and Khanna (1994) proved that the discrete-time models converge to the corresponding continuous-time models. This is important, as it provides a roadmap for establishing benchmarks. A number of variants of the original CRR trees have been proposed. Boyle (1986) proposed the trinomial tree in which each node generates three branches. Leisen and Reimer (1996, LR) and Tian (1993, Tian) reconstructed the CRR binomial model by modifying the effect of parameters on jumps between nodes and probabilities. The adaptive mesh method proposed in Figlewski and Gao (1999) embedded a secondary finer lattice mesh in key parts of the grid, which dramatically reduces the nonlinear error and has a wide range of applications. Staunton (2005) improved the LR model using Curtailed Ranges and Richardson extrapolation. The revamped LR model is the most efficient compared with the two best base approximations. Joshi (2009) and Chen and Joshi (2012) accelerated the Tian model using truncation, smoothing and Richardson extrapolation, which produce better performance than the improved LR model. Shang and Byrne (2019) proposed an accelerated CRR model incorporating an intelligent lattice search algorithm, truncation and dynamic memory technologies. This model proved to be more efficient than the best performing model proposed by Chen and Joshi (2012), one of the leading benchmarks for lattice estimation, while did not in every instance surpass the closed form solution performance for American Option valuation. ${ }^{1}$ In this paper, we improve the LR and Tian model both in speed and accuracy with intelligent lattice search, acceleration technologies and accuracy modifications. We also show that the improved LR and Tian model can be more efficient than the Shang and Byrne (2019) tree and more efficient than leading closed formed solutions.

Identification of the optimal exercise boundary plays a central role in American option pricing. The optimal exercise boundary problem also exists for lattice models. Kim and Byun (1994) demonstrated

\footnotetext{
${ }^{1}$ In that paper, we used VBA to test and develop our models so that algorithms could be easily ported to any other desktop and peers could reproduce our research/results. In this paper, we rely upon $\mathrm{C}++$ which tends on the whole to be more efficient that VBA. It also tends to influence the relative performance/efficiency of proposed models.
} 
the properties associated with the optimal exercise boundary of a CRR binomial model for an American put option without dividends payment. A definable locus of nodes that map out the stopping and continuation regions of the binomial tree can be revealed. Curran (1995) further extended the KimByun boundary theory for American options with continuous dividend yields. Curran's boundary theory however is constrained in that the risk-free interest rate, $r$ is necessarily superior (inferior) to the dividend yield, y for the put (call) option. Shang and Byrne (2019) overcome that limitation by initiating backward recursion from the penultimate column. This relatively small tweak implies that Curran's boundary theory can be relaxed to include a wider subset of parameter inputs for CRR. Basso et al. $(2002,2004)$ developed a binomial approximation to the optimal exercise boundary with the insight of Kim-Byun boundary theory. Areal and Rodrigues (2013) extended Curran's boundary theory to accelerate the binomial lattice for valuing American options with discrete dividends. Guo and Liu (2019) further proposed a binomial model for pricing options with known dollar dividends. In this paper, we extend the insights developed in Shang and Byrne (2019) and apply intelligent lattice search insights to both the LR and Tian trees with unrestricted continuous dividend yield.

In order to test and compare the efficiency of option pricing models, we need to amply generate random samples of option parameters. Broadie and Detemple (1996) set out criterion for producing random parameters, which has been adapted in turn by Chen and Joshi (2012) and Fabozzi, et al (2016). Gaudenzi and Pressacco (2003) and Pressacco, et al (2008) however found that the accuracy of American option pricing is linked to strong/weak underlying condition of parameters. Strong options, while few relative to all options, contribute proportionately the largest part of the estimation error. In this paper, we generate parameter samples following Broadie and Detemple (1996) and then filter results in terms of moneyness and the ratio of American to European (AER) analogues to signal strong/weak. ${ }^{2}$

The remaining paper is organized as follows. In Section 2, we introduce the LR tree and Tian tree and their optimal exercise boundary theory. In Section 3, we discuss two acceleration technologies and two accuracy modifications which can be used to improve LR and Tian models in speed and accuracy respectively. In Section 4, we demonstrate the pricing process of the improved LR and Tian models. In Section 5, we compare a series of improved LR and Tian models with the leading benchmark tree and more importantly to well-known analytical formulae. In each instance, we follow Strong-Weak-In-Out filtering consistent Pressacco, et al (2008). In Section 6, we conclude.

\footnotetext{
${ }^{2}$ Pressacco et al. (2008) chose an AER ratio of 1.4 to filter options in terms of being strong. They noted that large and erratic errors are common in a number of techniques that purported to value American options when AER exceeds 1.4.
} 


\section{LR and Tian Trees and their Optimal Exercise Boundary Condition}

Consider an American put option with spot price $\mathrm{S}$, strike price X, time to maturity $\mathrm{T}$, risk-free interest rate $r$, continuous dividend yield $y$, volatility $\sigma$, and a step size $n$. The time step is $\Delta t=T / n$. Leisen and Reimer (1996) proposed an optimized binomial tree that centres around the exercise price using PreizerPratt inversion methods. They define the probability of moving upward and downward, $\mathrm{p}$ and $\mathrm{q}$ respectively, and the magnitude of upward and downward factors $\mathrm{u}$ and $\mathrm{d}$, as:

$$
\begin{gathered}
p=h\left(d_{2}\right) \\
q=1-p \\
u=e^{(r-y) \Delta t} \frac{h\left(d_{1}\right)}{h\left(d_{2}\right)} \\
d=\frac{e^{(r-y) \Delta t}-p u}{1-p}
\end{gathered}
$$

where

$$
\begin{gathered}
d_{1}=\frac{\ln \left(\frac{S}{x}\right)+\left(r-y+\frac{\sigma^{2}}{2}\right) T}{\sigma \sqrt{T}} \\
d_{2}=d_{1}-\sigma \sqrt{T} \\
h(x)=\frac{1}{2}+\frac{\operatorname{sgn}\left(d_{1}\right)}{2} \sqrt{1-\exp \left[-\left(\frac{x}{n+\frac{1}{3}}\right)^{2}\left(n+\frac{1}{6}\right)\right]}
\end{gathered}
$$

$\operatorname{Sgn}()$ is the sign function and the number of time steps $n$ should be odd. ${ }^{3}$ Tian (1993) proposed that the binomial parameters is selected such that discrete-time distribution converges to the continuous-time distribution. Tian (1993) stipulated that $\mathrm{p}, \mathrm{q}, \mathrm{u}$, and $\mathrm{d}$ are defined as:

$$
\begin{gathered}
\mathrm{p}=\frac{M-d}{u-d} \\
\mathrm{q}=1-\mathrm{p}=\frac{u-M}{u-d} \\
\mathrm{u}=\frac{M V}{2}\left[(V+1)+\sqrt{V^{2}+2 V-3}\right] \\
\mathrm{d}=\frac{M V}{2}\left[(V+1)-\sqrt{V^{2}+2 V-3}\right]
\end{gathered}
$$

where $M=\exp (r \Delta t)$ and $V=\exp \left(\sigma^{2} \Delta t\right)$. Other than the varying definition of $u, d$ and $p$, the stock price tree adheres to the same construction. Likewise, the backward induction method relevant for LR and Tian model is performed in the usual fashion, not unlike, the conventional CRR binomial tree. Each node in the binomial tree can be identified as having a $(i, j)$ mapping, where $i$ represents the $i^{\text {th }}$ column in which the node is located and $j$ represents the number of upward movements of stock price consistent with each node. The maximum $j$ is determined by the $\mathrm{i}^{\text {th }}$ column count. The option value of node $(i, j)$ is represented as $\mathrm{V}(i, j)$. Since the LR and Tian binomial tree uses a backward inductive pricing process,

\footnotetext{
${ }^{3}$ Here we calculate $\mathrm{h}(\mathrm{x})$ using the Preizer-Pratt inversion method 2. Alternatively, Preizer-Pratt inversion method 1 can also be used.
} 
the pricing starts from the nodes at the maturity. At the final column (expiry), the respective put option values of the nodes are determined by:

$$
V_{(n, j)}=\max \left(S u^{j} d^{n-j}-X, 0\right)
$$

From the penultimate column back, the option values of the nodes are equal to the maximum of the exercise value or the holding value which is defined as:

$$
V_{(i, j)}=\max \left\{S u^{j} d^{i-j}-X,\left[p V_{(i+1, j+1)}+(1-p) V_{(i+1, j)}\right] / R\right\}
$$

where $\mathrm{R}=\exp (\mathrm{r} \Delta \mathrm{t})$. The exercise value equates to the difference between the stock price of the node $(i, j)$ and the fixed strike price $\mathrm{X}$. The holding value is defined as the discounted weighted-average sumproduct of two nodes from the preceding column, node $(i+1, j+l)$ and node $(i+1, j)$.

Kim and Byun (1994) developed some definitions/nomenclature for pricing non-dividend paying American put options using a CRR lattice. We extend these naming and mapping conventions to the LR and Tian binomial trees. A binomial tree can be divided into two regions: the stopping region $S$ where the option is exercised and a continuation region $\boldsymbol{C}$ where the option is held. Formally, two regions are defined as:

$$
\begin{aligned}
& \boldsymbol{S} \equiv\left\{(i, j) \mid V_{(i, j)}=X-S_{(i, j)}\right\} \\
& \boldsymbol{C} \equiv\left\{(i, j) \mid V_{(i, j)}>X-S_{(i, j)}\right\}
\end{aligned}
$$

The nodes in the stopping region are called stopping nodes and their values are equal to their corresponding exercise values. Similarly, the nodes pertaining to the continuation region are referred to as continuation nodes and equate to holding values. The optimal exercise node is the last stopping node which delineates the stopping nodes from the continuation nodes for each column. The nodes on and above the optimal exercise node are stopping nodes while the nodes below are continuation nodes. Optimal exercise nodes at each column constitute the optimal exercise boundary which separates the stopping and continuation regions. Following Kim and Byun (1994) and Curran (1995), Shang and Byrne (2019) proposed an extended optimal exercise boundary theory that draws from efficiently delineating the stopping and holding regions. We found that the intelligent lattice search algorithm can be applied to LR and Tian trees. As before: the optimal exercise boundary of a LR and Tian binomial tree is continuous from the penultimate column back when pricing American options. Consistent with Shang and Byrne (2019) no restrictions must be imposed on dividend yield relative to the risk-free rate. The simplified numerical examples in Fig. 1 can be used to demonstrate some properties of the optimal exercise boundary for a LR and Tian tree, where it is noted that the boundary is continuous when implementing backward recursion from the penultimate column. Consider an American put option with the following parameter values: $S=100, X=100, T=1, r=0.05, y=0.07^{4}$ and $\sigma=0.3$. The 9-step

\footnotetext{
${ }^{4} \mathrm{y}>\mathrm{r}$ which violates the assumption set out by Curran (1995). $\mathrm{y}=0.07$ is deliberately engineered to exceed $\mathrm{r}$.
} 
LR and Tian binomial trees for pricing this option are shown in Fig. $1 .{ }^{5}$ Each node is represented by its corresponding option value. Importantly, the nodes in each column are organised in descending values for options which implies the stock price tree is flipped. The values marked in bold represent stopping nodes and the rest of values that are not marked in bold constitute continuation nodes. $(i$,$) and (, j)$ signify the column and row number of the node respectively, where $i$ and $j=0,1, \ldots 9$. The nodes falling on the same horizontal (tilted) light dashed line have the same column (row) number. The optimal exercise nodes are underlined and connected by the heavy lines: marking the optimal exercise boundary. As with Shang and Byrne (2019), it is clear that the boundary is continuous from the penultimate column stretching backward. In contrast, between the final and penultimate column, continuity cannot be guaranteed. Here, the solid continuous locus is interrupted by a jump between non-adjacent nodes marked by a heavy dashed line. The continuous boundary indicates that the $j$ value (row number) associated with the optimal exercise node at each column and the previous column, are either equal or differ by a value of 1 . In other words, if the optimal exercise node at $i^{\text {th }}$ column is known as $(i, j)$, the optimal exercise node at $(i-1)^{\text {th }}$ column is either $(i-1, j)$ or $(i-1, j-1)$. Therefore, the optimal exercise boundary can be confirmed by verifying the exercise condition of merely a single node in each column. ${ }^{6}$ Once the boundary is determined, stopping and continuation regions/nodes can be further determined without any additional checks. Using the optimal exercise boundary theory, disposes of the requirement for costly blanket inspection to determine the exercise condition of each node. This permits an efficient bifurcation of the tree between stopping and continuation regions and dramatically reduces computational costs.

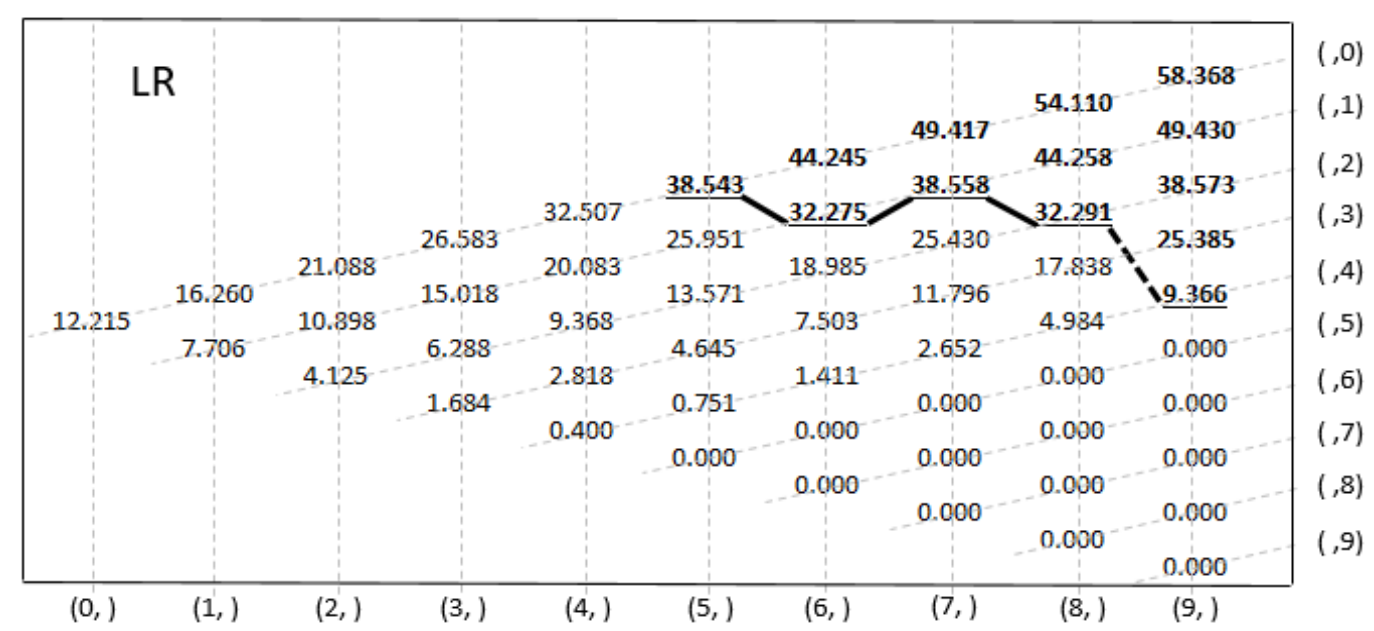

\footnotetext{
${ }^{5}$ A step-size of 9 adheres to the LR stipulation that $n$ should be odd. In the algorithm we developed, if $n$ happens to be even then the step sizes defaults to $\mathrm{n}+1$.

${ }^{6}$ Conventional trees require a blanket search.
} 


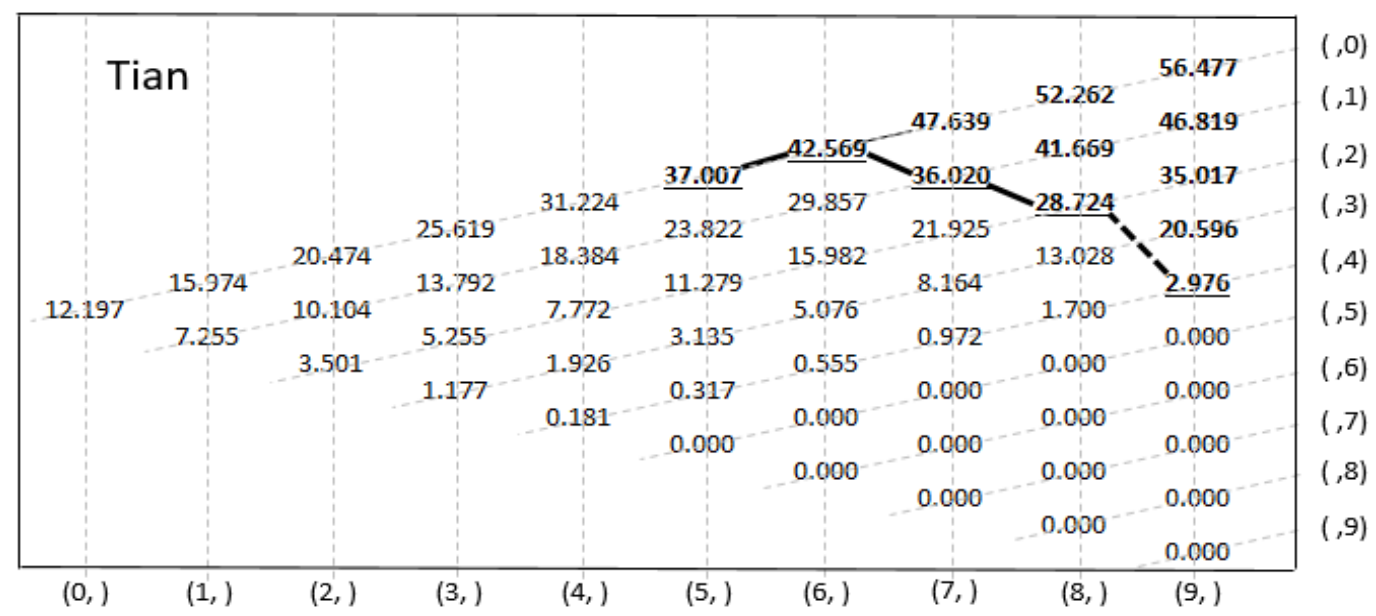

Fig. 1 Optimal exercise boundary of a LR and Tian tree

\section{Acceleration Technologies and Accuracy Modifications}

In this section, we introduce two acceleration technologies relating to truncation and dynamic memory and two accuracy modifications relating to Black-Scholes modification and Richardson Extrapolation. The latter two were proposed by Broadie and Detemple (1996). Truncation eliminates nodes that exert no effects on backward recursion but otherwise are nearly as computationally expensive as relevant nodes. Truncation purges nodes with a value of zero and stopping notes beyond the optimal exercise boundary. The dynamic approach releases memory because it is not necessary to store the entire tree at once. These two acceleration technologies, as well as the optimal exercise boundary theory, accelerate the computation but do not impact on accuracy. The accelerated trees can be made to replicate the original estimations but with a much higher speed. In contrast, accuracy modifications focus on improving the pricing accuracy while the speed is reduced marginally. Broadie and Detemple (1996) explained how the Black-Scholes formula can be introduced into the lattice at the $(n-1)^{\text {th }}$ time step (penultimate column). This was coupled with Richardson Extrapolation which improves efficiency, in principle, "by extrapolating to the limit". In principle, one might expect that numerical models adapted using Black-Scholes modification and/or Richardson Extrapolation have higher accuracy than their original configurations. Interestingly, we find that Intelligent Lattice Search can be combined with truncation and dynamic memory. This combination is developed to configure the accelerated model. Models that apply Black-Scholes modification or Richardson Extrapolation bear the suffix "BS" or "RE" or "BS\&RE".

When we price a European option using an original unmodified LR or Tian binomial tree, the option value of the node in one column is always generated from two nodes in the preceding column. ${ }^{8}$ Therefore, through the backward induction pricing process, all nodes in the tree partially contribute to

\footnotetext{
${ }^{7}$ Broadie and Detemple (1996) refer to the combined approach as "BBRS".

${ }^{8}$ The exception being at maturity.
} 
the generation of the apex node or present value of option. As noted previously for American lattices in (13), each node from the penultimate column back is equal to the maximum of the exercise value or the holding value. ${ }^{9}$ If the holding value is greater than the exercise value, the former will be assigned to the node. Otherwise, the option's value is equal to the exercise value which is the difference between the fixed strike price and the corresponding stock price of the node. In this event, the two adjacent nodes in the preceding column do not influence any value in the backward induction pricing process. This leads to a powerful conclusion. The value of an American option is only dependent on nodes within specific regions of the LR binomial tree. Curran (1995) proposes dividing the CRR lattice into subtrees, where some nodes exert no influence on the present value of the option but other nodes do. In this section, we apply the same truncation technology to LR and Tian binomial models and by extension prune the redundant nodes. In Fig. 2, we take an example of the same 9-step LR binomial tree as Fig. 1. Each number represents the option value of each node for this binomial tree. The values marked in bold are exercise values and the rest (not marked in bold) are holding values. The arrows connecting two option values represent the value-delivering process. It is not hard to observe that the apex node at the first column is a repository that contains information filtered from the entire LR binomial tree. There are some redundant nodes, enclosed in the two triangular regions, which are not involved in the valuedelivering process. The nodes associated with the upper triangle do not influence the "next generation", which means their option values cannot be transferred to the next column and thereafter. For the lower triangle, the option values of nodes are zero. Truncation is typically implemented by recognising the redundant regions and excluding them algorithmically to reduce the computational load.

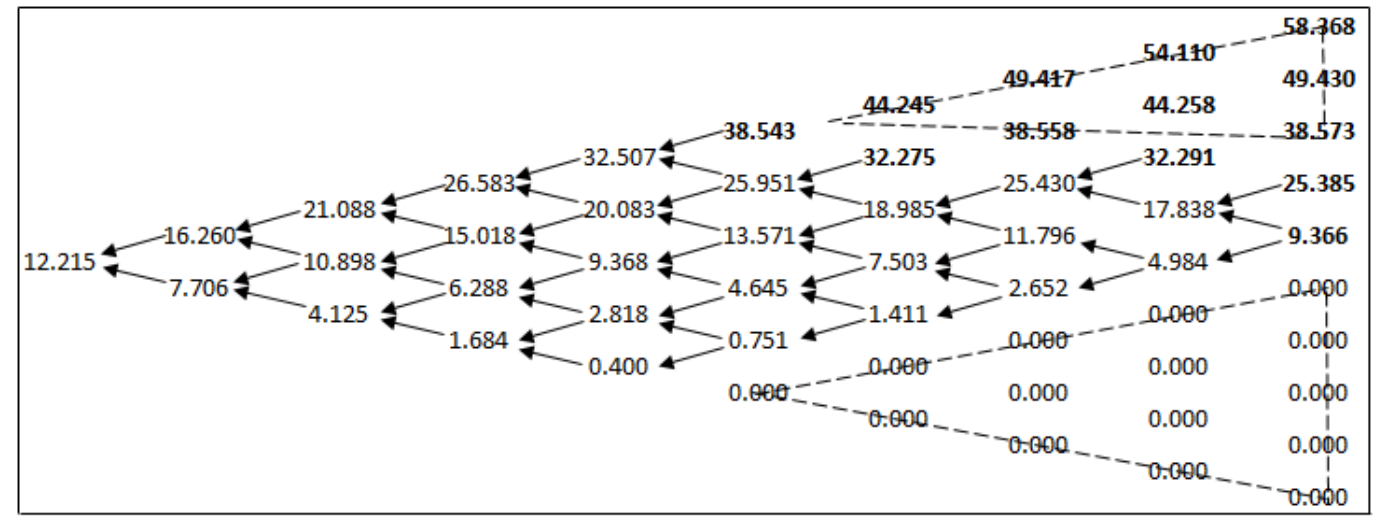

Fig. 2 Truncation technology

In a static memory environment, spaces are allocated in preparation for memorizing each option value computed in the valuation process. For a n-step conventional binomial tree, the option values of $(n+1)(n+2) / 2$ nodes have to be memorized so that an appropriate quantity of static memory spaces are

\footnotetext{
${ }^{9}$ The holding value is derived from two nodes in the previous column.
} 
assigned $^{10}$. Broadie and Detemple (1996) and Haug (1997) propose a dynamic memory method. For either a n-step dynamic LR or Tian binomial tree, only a maximum of $(n+1)$ memory spaces need to be allocated and option values of $(n+1)$ nodes need to be memorized sequentially. Using dynamic memory, the backward inductive pricing is also a substitution process where option values previously stored in the memory spaces are replaced by newly generated option values. In Fig. 3, we construct a 5-step dynamic LR binomial tree for pricing the same American put option as Fig. 1. Here we use $(j)$ instead of $(i, j)$ to locate the node at each column, where $j=0,1, \ldots, 5$. The nodes falling on the same slant dashed line (upper figure) share the same serial number $j$. In the dynamic substitution process (lower figure), we first allocate six memory spaces, $V(0)-V(5)$, represented by rectangles, in which the computed option values of nodes at the last column are stored. When we move backward to the penultimate column, the option values at this column are computed and replace option values previously stored in $\mathrm{V}(0)-\mathrm{V}(4)$. Similarly, option values at antepenultimate column are computed and replace values previously memorized in $\mathrm{V}(0)-\mathrm{V}(3)$. This computation and substitution process lasts until the option value at the first column is computed and memorized in $\mathrm{V}(0)$. Dynamic memory conserves precious processing power by virtue that this style of implementation simply employs $O(n)$ storage. Conventional static lattice implementations, with n steps, possess $O\left(n^{2}\right)$ nodes and consequently computation time increases in tandem with $O\left(n^{2}\right)$.

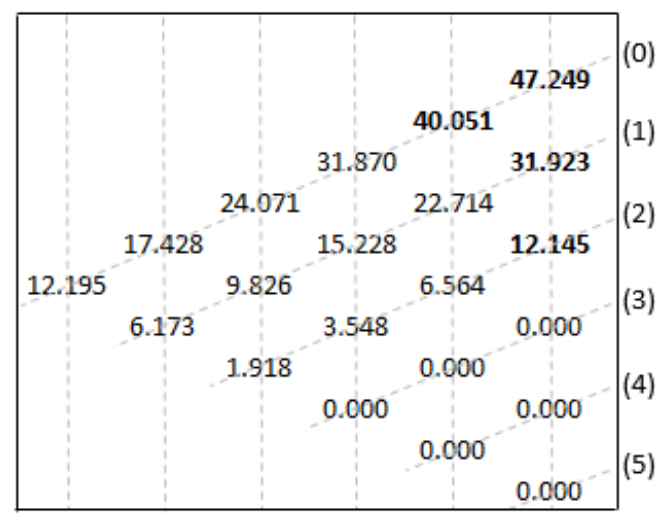

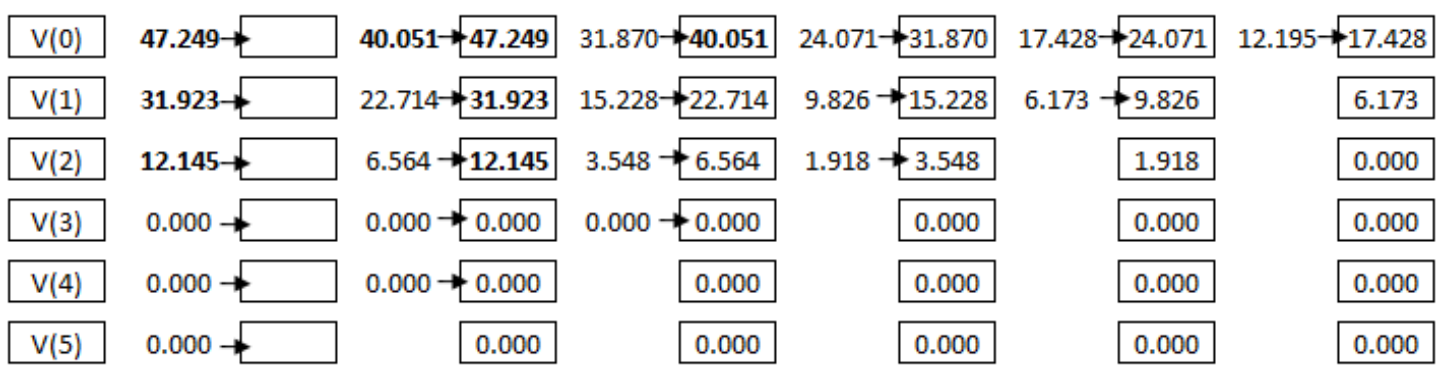

Fig. 3 Dynamic memory

\footnotetext{
${ }^{10}$ For example, a 2 -step binomial tree has 6 nodes with option values $\mathrm{V}(0,0), \mathrm{V}(1,0), \mathrm{V}(1,1), \mathrm{V}(2,0), \mathrm{V}(2,1)$, $\mathrm{V}(2,2)$ respectively so that 6 static memory spaces are allocated.
} 
Broadie and Detemple (1996) introduce two modifications to the binomial method: Black-Scholes continuity values at the penultimate nodes and Richardson extrapolation. In principle, the Black-Scholes modification improves the binomial tree by replacing the conventional holding values of each node at the penultimate column with the corresponding set of Black-Scholes values. The option value of the nodes at the penultimate column is given by:

$$
V_{(n-1, j)}=\max \left\{X-S u^{j} d^{(n-1)-j}, B S\left(S u^{j} d^{(n-1)-j}, X, \Delta t, r, y, \sigma\right)\right\}
$$

Where BS(.) represents the Black-Scholes value. Since the Black-Scholes model is originally designed for pricing European options at the limit, more accurate holding values can be obtained. It should be noted, of course, that when applying Black-Scholes valuation, the zero-value nodes will be replaced by non-zero Black-Scholes values. This impedes the truncation of the zero-value zone and imposes an additional computational workload.

Broadie and Detemple (1996) shows that the accuracy of a binomial model can be significantly improved by using two-point Richardson Extrapolation. Allow $\mathrm{f}(\mathrm{n})$ to denote the estimation of an option price using a $n$-steps binomial mode ${ }^{11}$. The extrapolated option price, $f_{R E}(n)$, is easily obtained by:

$$
f_{R E}(n)=2 f(n)-f\left(\frac{n}{2}\right)
$$

Unlike other technologies, Richardson Extrapolation will be applied only after the option prices have been obtained from two binomial trees with $\mathrm{n}$ and a half-n number of steps. Richardson Extrapolation can be easily adapted to a lattice model but does impose added computational costs. Joshi (2009) and Chen and Joshi (2012) noted that the Black-Sholes modification interacts well with Richardson Extrapolation. The combination of these two modifications significantly improves the accuracy but only marginally decreases the speed within conventional lattice structures.

\section{Applying Truncation, Dynamic Memory and Intelligent Lattice Search to LR and Tian}

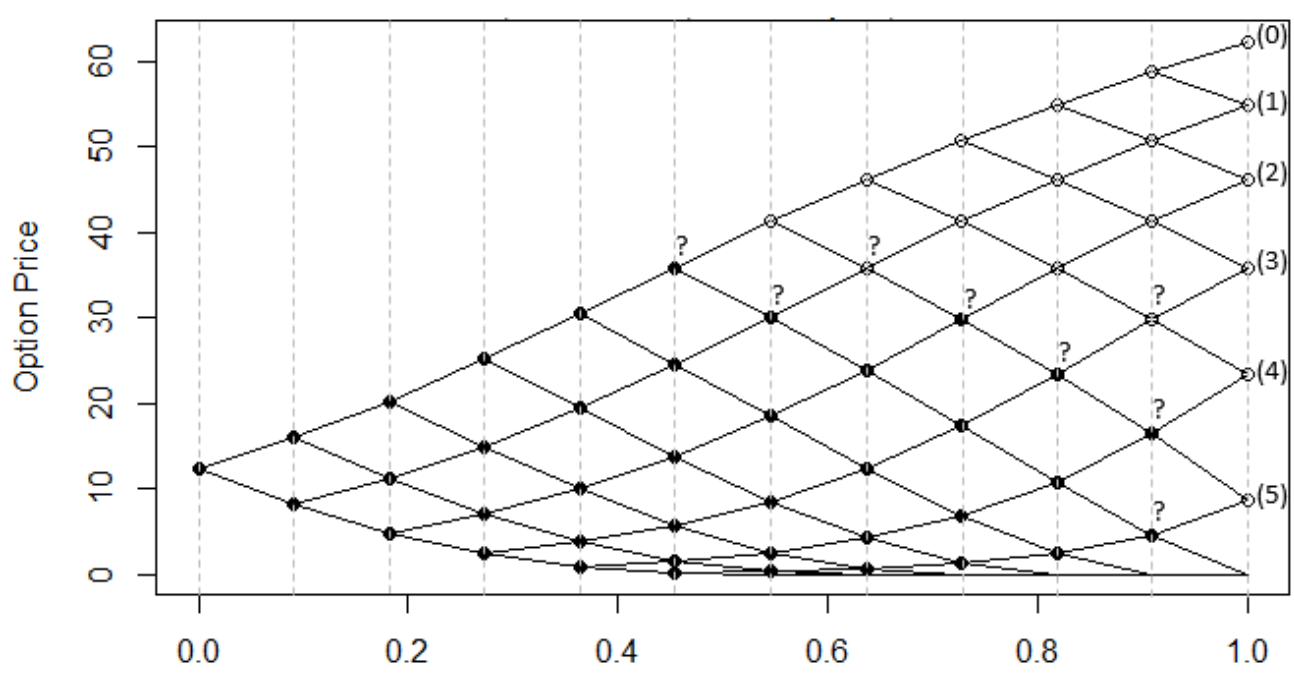

Binomial Period

\footnotetext{
${ }^{11}$ Here we assume $\mathrm{n}$ is even.
} 
Fig. 4 Pricing an American option using an accelerated LR binomial model

In this section, we tease out the pricing process of both the accelerated LR binomial model and accelerated Tian model. The original LR and Tian model can be accelerated by using intelligent lattice search, dynamic memory, and truncation technologies. The accelerated pricing process will be presented first and then the work-flow logic outlined in Fig. 4 will be described. Consider the same American put option as identified in Fig. 1 where $S=100, X=100, T=1, r=0.05, y=0.07$ and $\sigma=$ 0.3. An 11-step ${ }^{12}$ LR binomial tree for pricing this option is shown in Fig. 4. The $\mathrm{X}$ axis represents the binomial period. The $\mathrm{Y}$ axis represents the option price so that the zero-value region of the tree naturally disappears. The serial number from (1) to (5) correspond to each upward curve of the tree. The nodes falling on the same upward curve have the same serial number. The vertical dashed lines represent each column. Solid and hollow nodes represent the continuation and stopping nodes respectively. Only the nodes with a question mark above, also called uncertain nodes, need to be inspected to verify the exercise condition.

The backward inductive option pricing starts from the maturity (the final column). An interesting feature of a LR binomial tree relates to the first non-zero value node or node (h) at each step, where $h$ $=(\mathrm{n}-1) / 2$. In other words, the nodes in the LR tree with a serial number greater than $\mathrm{h}$ are all zerovalue redundant nodes, which can be easily recognised and truncated. The Tian tree, however, does not possess this feature so that the exercise condition of the nodes at the maturity has to be checked one by one until the first non-zero-value node is determined. In Fig. 4, the first non-zero value node at the last column of the tree is node (5) as $h=5$, which is also the optimal exercise node (first stopping node) at that column. The corresponding exercise values, from 62.22 to 8.54 , should be computed and assigned to nodes (0) - (5) in the final column. The nodes below node (5) are recognized and truncated as zerovalue nodes.

Next, we move backward to the penultimate column. The optimal exercise boundary of the American put is not continuous between the last and penultimate column given that $r<y$. We therefore have to verify the exercise condition of each node in the penultimate column. We start from the first non-zerovalue node (h) in this column, comparing the holding value and exercise value of each node, until the optimal exercise node is found. Once the optimal exercise node is determined, we can cease iteratively verifying the exercise condition and start value assignment. The exercise values of the optimal exercise node, and the holding values of the nodes between the optimal exercise node and node (h), are then computed. ${ }^{13}$ The remaining nodes in this column can then be truncated as redundant nodes. In Fig. 4, at the penultimate column, we examine the exercise condition of each node, starting from node (5) until node (3) which is confirmed as the optimal exercise node. Then the exercise value of 29.85 is computed

\footnotetext{
${ }^{12}$ An odd number of steps is required for option pricing in a LR binomial model.

${ }^{13}$ The nodes between the optimal exercise nodes and node (h) include node (h) but exclude optimal exercise node.
} 
and assigned to node (3), and the holding value, 16.35 and 4.52, are calculated and assigned to nodes (4) and (5).

From the penultimate column back, we are able to apply intelligent search and avoid a blanket inspection of the exercise-condition. For the remaining columns, the exercise condition of only one uncertain node needs to be examined - the node with the same serial number as the optimal exercise node of the previous column. ${ }^{14}$ There are two possible outcomes for the uncertain node at each column:

(1) If its exercise value is greater than the holding value, this node is the optimal exercise node at this column. The corresponding exercise values are calculated and assigned to the optimal exercise node and the node immediately above it.

(2) If its exercise value is smaller than holding value, this implies that the node is a continuation node and the node immediately above it should be the optimal exercise node at the same column. The corresponding exercise values are calculated and assigned to the optimal exercise node.

The holding values are computed and assigned to the nodes between the optimal exercise node and node (h). The remaining nodes at that column: the zero-value nodes and the redundant stopping nodes are truncated. This efficient pricing process can be applied to the antepenultimate column and each column thereafter. In Fig. 4, when we move to the antepenultimate column, only the exercise condition of node (3) needs to be verified since the optimal exercise node at the penultimate column has the serial number of 3. Once node (3) is determined as a continuation node (holding value > exercise value), the optimal exercise node at the antepenultimate column should be node (2). Then the exercise value 35.78 is assigned to node (2) and the holding values 23.42, 10.77 and 2.40 are assigned to node (3)-(5) respectively. The rest of nodes at this column can be truncated.

This efficient pricing process is further applied to each of the remaining columns until the column at which all nodes are stopping nodes (or continuation nodes), which indicates that all nodes in the remaining columns are stopping nodes (or continuation nodes). If the remaining nodes are all stopping nodes, the present value of the option can be directly equal to the difference between strike price and spot price. Otherwise, if they are all continuation nodes, the holding value of each node in the rest of columns are computed to obtain the present value of the option. In Fig. 4, the efficient backward inductive pricing process ends at the column fully consisting of solid nodes (continuation nodes). Hence, the corresponding holding value are computed and assigned to the nodes at each of the other columns. Finally, the present value of the option is obtained as 12.22 .

Combining intelligent lattice search, dynamic memory and truncation conspires to dramatically accelerate the computation speed for the LR binomial model. Instead of examining the exercise

\footnotetext{
${ }^{14}$ The optimal exercise boundary theory implies that if the optimal exercise node at $i^{\text {th }}$ column is node $(j)$, the optimal exercise node at $(\mathrm{i}-1)^{\text {th }}$ column has only two possibilities: either node $(j)$ or node $(j-1)$.
} 
condition of each node of the tree, we only need to inspect at most one uncertain node in each column from the antepenultimate column back. Furthermore, redundant zero-value nodes and redundant stopping nodes can also be easily recognised and truncated. Moreover, this algorithm saves significant memory spaces by virtue that only $(h+1)$ instead of $(n+1)(n+2) / 2$ memory space is required.

Both the accelerated LRBS and TianBS models apply Black Scholes smoothing in the accelerated LR and Tian models. This entails making a number of changes. All nodes at the last column are discarded because they will not infer their values backward. The pricing process starts from the penultimate column, where we verify the exercise condition of each node by comparing the Black-Scholes value with the corresponding exercise value. This evaluation ends once the optimal exercise node is determined where the exercise value is greater than the Black-Scholes value. Then the corresponding exercise values are assigned to the optimal exercise node and the corresponding Black-Scholes values are assigned to the nodes below the optimal exercise node. From the antepenultimate column back, the pricing process of the accelerated LRBS and TianBS trees are the same as the accelerated LR and Tian tree. $^{15}$

\section{Numerical Results}

In this section, we assert that the standard LR and Tian model can be effectively accelerated by adapting intelligent lattice search, truncation and dynamic memory without disturbing the accuracy of the parent model. We shall compare variants of the accelerated LR and Tian models with commonly used analytical formulae for pricing American options. We make a similar comparison to leading benchmark lattice, based on randomly sampled parameters. Finally, we divide sample options into four groups according to the AER ratio and the moneyness of the options. We test the relative performance of the efficient LR and Tian models based on intelligent lattice search for varying option specification.

As with previous authors, we need an error measure and time consumption measure that go beyond purely convergence and step size limited to one set of parameters with an incremental step size. In addition, we need a sufficient level of randomization, so our results are not the product of any specific pattern in the choice of parameters or reflecting other biases. To meet this challenge, we follow Broadie and Detemple (1996) and generate 2,500 American option parameter combinations. The stock price S is distributed uniformly between 70 and 130 . Time to maturity $\mathrm{T}$ is also uniformly randomised with boundaries between 0.1 and 1 years with a probability weight of 0.75 and additionally 1 to 5 years with a probability weighting of 0.25 . The risk-free interest rate $r$ is bounded between 0 and 0.1 with probability of 0.8 and equal to 0 with a probability of 0.2 . The dividend yield, y, is uniformly generated spanning 0 to 0.1 . Volatility $\sigma$ ranges between 0.1 and 0.6 and adheres to a uniform distribution. The strike price $\mathrm{X}$ is fixed at 100 . The options are configured with a 0.5 probability of being a call or a put.

\footnotetext{
${ }^{15}$ Notice that for an accelerated LRBS and TianBS tree, the zero-value zone disappears and is replaced by nonzero Black-Scholes values.
} 
The error measure, root mean squared relative error (RMSRE), is defined consistently with Broadie and Detemple (1996) as:

$$
\operatorname{RMSRE}=\sqrt{\frac{1}{m} \sum_{i=1}^{m} e_{i}^{2}}
$$

where $m=$ the number of American options and $e_{i}=\frac{\widehat{c}_{l}-C_{i}}{C_{i}}$ with $\widehat{C}_{l}$ representing the estimation of the option value and $C_{i}$ representing the true value of the option generated by a 15,000-step CRR binomial model. We discard 170 options with true values smaller than 0.5. Finally, 2,330 American options are contained in the valuation. The time consumption measure, average CPU time (Time), is defined as:

$$
\text { CPU time }=\frac{\text { Sum of Processsor Time }}{2330}
$$

All the numerical results are generated in C++ Visual Studio 2015 on a DELL Latitude E5470 laptop with Intel Core i3-6100U CPU (2.30 GHz) and 8GB of RAM.

Initially we demonstrate that intelligent lattice search, truncation and dynamic memory technologies unambiguously accelerate the LR and Tian models without altering original estimates. We price 2,330 American options generated above using both an accelerated LR (Accel LR) and a standard LR (LR) model and using both an accelerated Tian (Accel Tian) and a standard Tian (Tian) model. The number of steps for all pairings range from 100 to 1500 in intervals of $100 .{ }^{16}$ The corresponding RMSRE and CPU Time are reported in Table 1, where the LR and Accel LR always have identical RMSRE and likewise Tian and Accel Tian always correspondingly have identical RMSRE but the accelerated versions takes much less computational time than the conventional analogues. In Fig. 5, we graph the results associated with LR models in Table 1 to make it more intuitive. Two solid curves represent the Accel LR and LR respectively. Each point represents a combination of RMSRE and Time generated by the corresponding tree. It is noteworthy that the Accel LR and LR options sharing the same number of steps share also the same reported RMSRE. This provides some evidence that the Accel LR and LR produce exactly the same estimations. ${ }^{17}$ The length of the vertical dashed lines separating the CPU Time for the Accel LR arc and the LR arc in Fig. 5, points to inherent efficiencies in respect to the Accelerated model. This increases exponentially as the number of steps are increased. This improvement in efficiency can be explored further by introducing modifications for Black Scholes smoothing and

\begin{tabular}{|c|c|c|c|c|c|c|c|c|}
\hline \multirow[b]{2}{*}{ Steps } & \multicolumn{2}{|c|}{ Accel LR } & \multicolumn{2}{|c|}{ LR } & \multicolumn{2}{|c|}{ Accel Tian } & \multicolumn{2}{|c|}{ Tian } \\
\hline & RMSRE & Time & RMSRE & Time & RMSRE & Time & RMSRE & Time \\
\hline 100 & $0.0562 \%$ & $3.72 \mathrm{E}-03$ & $0.0562 \%$ & $1.24 \mathrm{E}-02$ & $0.389 \%$ & $3.71 \mathrm{E}-03$ & $0.389 \%$ & $1.79 \mathrm{E}-02$ \\
\hline 200 & $0.0285 \%$ & 7.49E-03 & $0.0285 \%$ & $5.76 \mathrm{E}-02$ & $0.187 \%$ & $1.09 \mathrm{E}-02$ & $0.187 \%$ & $5.71 \mathrm{E}-02$ \\
\hline 300 & $0.0193 \%$ & $1.59 \mathrm{E}-02$ & $0.0193 \%$ & $1.19 \mathrm{E}-01$ & $0.128 \%$ & $2.09 \mathrm{E}-02$ & $0.128 \%$ & $1.14 \mathrm{E}-01$ \\
\hline 400 & $0.0144 \%$ & $2.67 \mathrm{E}-02$ & $0.0144 \%$ & $1.83 \mathrm{E}-01$ & $0.095 \%$ & $3.49 \mathrm{E}-02$ & $0.095 \%$ & $1.94 \mathrm{E}-01$ \\
\hline
\end{tabular}
Richardson Extrapolation. These findings would appear also apply to the Accel Tian and Tian model.

\footnotetext{
${ }^{16}$ Notice that the actual number of steps that a series of LR models used is the step number shown on the table plus one since LR models require odd steps.

${ }^{17}$ No differences between either sets of options were found for the Accel LR and Conventional LR model.
} 


\begin{tabular}{ccccccccc}
500 & $0.0115 \%$ & $4.22 \mathrm{E}-02$ & $0.0115 \%$ & $2.65 \mathrm{E}-01$ & $0.074 \%$ & $5.11 \mathrm{E}-02$ & $0.074 \%$ & $2.97 \mathrm{E}-01$ \\
600 & $0.0096 \%$ & $5.69 \mathrm{E}-02$ & $0.0096 \%$ & $3.67 \mathrm{E}-01$ & $0.064 \%$ & $7.44 \mathrm{E}-02$ & $0.064 \%$ & $4.05 \mathrm{E}-01$ \\
700 & $0.0082 \%$ & $7.88 \mathrm{E}-02$ & $0.0082 \%$ & $5.13 \mathrm{E}-01$ & $0.056 \%$ & $9.67 \mathrm{E}-02$ & $0.056 \%$ & $5.36 \mathrm{E}-01$ \\
800 & $0.0072 \%$ & $9.86 \mathrm{E}-02$ & $0.0072 \%$ & $6.47 \mathrm{E}-01$ & $0.045 \%$ & $1.25 \mathrm{E}-01$ & $0.045 \%$ & $7.26 \mathrm{E}-01$ \\
900 & $0.0065 \%$ & $1.25 \mathrm{E}-01$ & $0.0065 \%$ & $8.90 \mathrm{E}-01$ & $0.045 \%$ & $1.57 \mathrm{E}-01$ & $0.045 \%$ & $9.30 \mathrm{E}-01$ \\
1000 & $0.0059 \%$ & $1.71 \mathrm{E}-01$ & $0.0059 \%$ & $1.01 \mathrm{E}+00$ & $0.041 \%$ & $1.91 \mathrm{E}-01$ & $0.041 \%$ & $1.11 \mathrm{E}+00$ \\
1100 & $0.0055 \%$ & $1.87 \mathrm{E}-01$ & $0.0055 \%$ & $1.23 \mathrm{E}+00$ & $0.038 \%$ & $2.33 \mathrm{E}-01$ & $0.038 \%$ & $1.34 \mathrm{E}+00$ \\
1200 & $0.0051 \%$ & $2.11 \mathrm{E}-01$ & $0.0051 \%$ & $1.45 \mathrm{E}+00$ & $0.030 \%$ & $2.76 \mathrm{E}-01$ & $0.030 \%$ & $1.71 \mathrm{E}+00$ \\
1300 & $0.0047 \%$ & $2.47 \mathrm{E}-01$ & $0.0047 \%$ & $1.78 \mathrm{E}+00$ & $0.028 \%$ & $3.55 \mathrm{E}-01$ & $0.028 \%$ & $1.89 \mathrm{E}+00$ \\
1400 & $0.0045 \%$ & $2.85 \mathrm{E}-01$ & $0.0045 \%$ & $2.00 \mathrm{E}+00$ & $0.027 \%$ & $3.96 \mathrm{E}-01$ & $0.027 \%$ & $2.21 \mathrm{E}+00$ \\
1500 & $0.0043 \%$ & $3.43 \mathrm{E}-01$ & $0.0043 \%$ & $2.35 \mathrm{E}+00$ & $0.025 \%$ & $4.42 \mathrm{E}-01$ & $0.025 \%$ & $2.53 \mathrm{E}+00$ \\
\hline
\end{tabular}

Table 1. Comparing Accel LR (Accel Tian) and Standard LR (Tian)

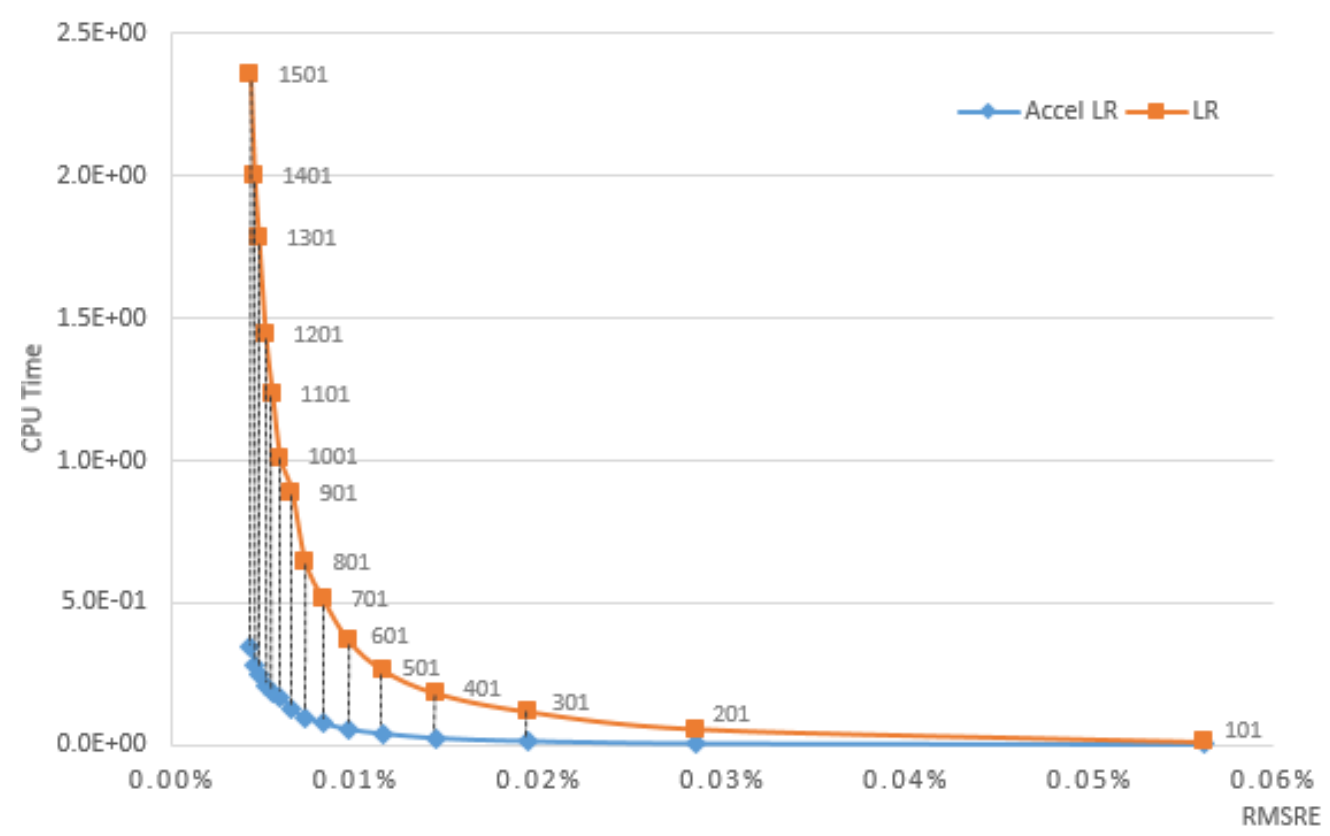

Fig. 5 Comparing the Accelerated LR and Standard LR models

In what follows, we compare the accelerated LR and Tian models adapted for different accuracy modifications with commonly used analytical formulae and the leading benchmark tree. These eight LR and Tian models include:

i. Accel LR: The accelerated LR model, which is the original LR model accelerated by using intelligent lattice search, dynamic memory, and truncation technologies

ii. Accel LRBS: Accel LR with Black-Scholes smoothing

iii. Accel LRRE: Accel LR with Richardson Extrapolation

iv. Accel LRBS\&RE: Accel LR with Black Scholes smoothing and Richardson Extrapolation

v. Accel Tian: The accelerated Tian model, which is the original Tian model accelerated by using intelligent lattice search, dynamic memory, and truncation technologies

vi. Accel TianBS: Accel Tian with Black-Scholes smoothing 
vii. Accel TianRE: Accel Tian with Richardson Extrapolation

viii. Accel TianBS\&RE: Accel Tian with Black Scholes smoothing and Richardson Extrapolation Importantly, as mentioned in the Introduction section, BAW, Bjerksund 93, Bjerksund 02, Ju-Zhong are four commonly used analytical formulae and the accelerated CRR model (Accel CRR) serves as a benchmark lattice model. We, initially, use relatively small step numbers ranging from 10 to 100 with increments of 10. In Table 2, we show the RMSRE and CPU Time when pricing 2,330 American options using eight LR and Tian models described above, the leading benchmark tree and four analytical formulae. It is obvious that each analytical formula can only provide one combination of accuracy and speed while lattice models more flexibly permit arbitrary levels of precision in tandem with a given tolerance for execution time. Fig. 6 allows us to visualize the results reported in Table 2, where the $\mathrm{X}$ (Y) axis represents RMSRE (CPU Time) respectively. In Fig. 6, lattice models are represented as curves and visualized in three sub-figures according to their performance relative to the benchmark analytical formulae. Four analytical formulae are represented by a single point separately in each of the three subfigures with the method name ascribed for clarity. We can mostly evaluate each model in terms of Pareto Improvement i.e. the arc (or point) closer to the origin that boasts the highest level of performance. It is discernible in the top-left sub-figure of Fig. 6 that the large segments of the two arcs embedded in the Accel LR and Accel LRRE loci are unmistakably closer to the origin relative to the four analytical formulae points, which indicates that Accel LR and Accel LRRE surpass analytical formulae in both speed and accuracy. In the top-right sub-figure, the Accel LRBS\&RE and Accel TianBS\&RE models perform better than three of the analytical formulae including Ju-Zhong, Bjerksund 02 and BAW although fare worse than Bjerksund 93. As evident in the bottom sub-figure, the rest of the lattice models, including Accel CRR, Accel LRBS, Accel Tian and Accel TianBS, have comparable performance relative to the analytical formulae. The four analytical formulae points largely coincide and are in the vicinity of these lattice models curves. Accel TianRE is not reported in Fig. 6 as performance proved to be inferior to the analytical formulae outlined in Table 2.

Higher accuracy can be achieved arbitrarily by increasing step size. From this vantage point, we proceed to compare eight accelerated LR and Tian models and the leading benchmark lattice model. We apply pricing to 2,330 American options using a relatively larger number of steps ranging from 100 to 1,000. Table 3 reports the corresponding RMSRE and CPU Time of these nine lattice models, which are graphed in Fig. 7. As before, the X (Y) axis represents RMSRE (CPU Time). It is evident that Accel TianBS\&RE, Accel LRRE and Accel LRBS\&RE boast the highest efficiency as their respective loci are closer to the origin. More specifically, the convergences of Accel TianBS\&RE is smoother and less noticeably oscillating relative to Accel LRRE and Accel LRBS\&RE. Accel LR also has the advantage of the smooth convergence but the efficiency is slightly lower than the best three. The secondary tier includes Accel LRBS, Accel CRR, Accel TianBS and Accel Tian, whose efficiency is one level lower than the previous four models. 


\begin{tabular}{|c|c|c|c|c|c|c|c|c|c|c|}
\hline \multirow{2}{*}{$\begin{array}{c}\text { Method } \\
\text { Steps } \\
\end{array}$} & \multicolumn{2}{|c|}{ Accel LR } & \multicolumn{2}{|c|}{ Accel LRBS } & \multicolumn{2}{|c|}{ Accel LRRE } & \multicolumn{2}{|c|}{ Accel LRBS\&RE } & \multicolumn{2}{|c|}{ BAW } \\
\hline & RMSRE & Time & RMSRE & Time & RMSRE & Time & RMSRE & Time & RMSRE & Time \\
\hline 11 & $0.5966 \%$ & $2.84 \mathrm{E}-03$ & $2.1327 \%$ & $2.93 \mathrm{E}-03$ & $0.6642 \%$ & $2.89 \mathrm{E}-03$ & $0.6817 \%$ & $3.02 \mathrm{E}-03$ & $1.24 \%$ & $3.17 \mathrm{E}-03$ \\
\hline 21 & $0.2783 \%$ & $2.92 \mathrm{E}-03$ & $1.1373 \%$ & $3.03 \mathrm{E}-03$ & $0.2282 \%$ & $2.94 \mathrm{E}-03$ & $0.2918 \%$ & $3.09 \mathrm{E}-03$ & \multicolumn{2}{|c|}{ Bjerksund93 } \\
\hline 31 & $0.1952 \%$ & $2.99 \mathrm{E}-03$ & $0.7764 \%$ & 3.07E-03 & $0.2481 \%$ & $3.03 \mathrm{E}-03$ & $0.2564 \%$ & $3.12 \mathrm{E}-03$ & RMSRE & Time \\
\hline 41 & $0.1434 \%$ & $3.00 \mathrm{E}-03$ & $0.5860 \%$ & $3.17 \mathrm{E}-03$ & $0.0774 \%$ & $3.05 \mathrm{E}-03$ & $0.0911 \%$ & $3.23 \mathrm{E}-03$ & $0.77 \%$ & $2.96 \mathrm{E}-03$ \\
\hline 51 & $0.1207 \%$ & $3.12 \mathrm{E}-03$ & $0.4726 \%$ & $3.26 \mathrm{E}-03$ & $0.0698 \%$ & $3.14 \mathrm{E}-03$ & $0.0768 \%$ & $3.32 \mathrm{E}-03$ & \multicolumn{2}{|c|}{ Bjerksund02 } \\
\hline 61 & $0.0946 \%$ & $3.13 \mathrm{E}-03$ & $0.3929 \%$ & $3.43 \mathrm{E}-03$ & $0.0440 \%$ & $3.19 \mathrm{E}-03$ & $0.0500 \%$ & $3.38 \mathrm{E}-03$ & RMSRE & Time \\
\hline 71 & $0.0771 \%$ & $3.25 \mathrm{E}-03$ & $0.3359 \%$ & $3.45 \mathrm{E}-03$ & $0.0343 \%$ & $3.27 \mathrm{E}-03$ & $0.0406 \%$ & $3.65 \mathrm{E}-03$ & $0.59 \%$ & $3.06 \mathrm{E}-03$ \\
\hline 81 & $0.0741 \%$ & $3.38 \mathrm{E}-03$ & $0.2958 \%$ & $3.51 \mathrm{E}-03$ & $0.0339 \%$ & $3.42 \mathrm{E}-03$ & $0.0364 \%$ & $4.18 \mathrm{E}-03$ & \multicolumn{2}{|c|}{ Ju-Zhong } \\
\hline 91 & $0.0665 \%$ & $3.55 \mathrm{E}-03$ & $0.2631 \%$ & $4.05 \mathrm{E}-03$ & $0.0337 \%$ & $3.70 \mathrm{E}-03$ & $0.0363 \%$ & $4.65 \mathrm{E}-03$ & RMSRE & Time \\
\hline 101 & $0.0562 \%$ & $3.72 \mathrm{E}-03$ & $0.2358 \%$ & $4.48 \mathrm{E}-03$ & $0.0312 \%$ & $3.99 \mathrm{E}-03$ & $0.0327 \%$ & $5.47 \mathrm{E}-03$ & $0.29 \%$ & $3.96 \mathrm{E}-03$ \\
\hline Method & \multicolumn{2}{|c|}{ Accel Tian } & \multicolumn{2}{|c|}{ Accel TianRE } & \multicolumn{2}{|c|}{ Accel TianBS } & \multicolumn{2}{|c|}{ Accel TianBS\&RE } & \multicolumn{2}{|c|}{ Accel CRR } \\
\hline Steps & RMSRE & Time & RMSRE & Time & RMSRE & Time & RMSRE & Time & RMSRE & Time \\
\hline 10 & $3.6776 \%$ & $2.80 \mathrm{E}-03$ & $5.3312 \%$ & $2.78 \mathrm{E}-03$ & $3.0778 \%$ & $3.00 \mathrm{E}-03$ & $1.1417 \%$ & $3.02 \mathrm{E}-03$ & $3.5614 \%$ & $2.90 \mathrm{E}-03$ \\
\hline 20 & $1.9925 \%$ & $3.29 \mathrm{E}-03$ & $3.1237 \%$ & $2.66 \mathrm{E}-03$ & $1.6298 \%$ & $2.87 \mathrm{E}-03$ & $0.2837 \%$ & $3.06 \mathrm{E}-03$ & $1.8507 \%$ & $2.95 \mathrm{E}-03$ \\
\hline 30 & $1.2083 \%$ & $2.86 \mathrm{E}-03$ & $1.8952 \%$ & $3.26 \mathrm{E}-03$ & $1.1000 \%$ & $3.29 \mathrm{E}-03$ & $0.1350 \%$ & $3.24 \mathrm{E}-03$ & $1.0773 \%$ & $2.96 \mathrm{E}-03$ \\
\hline 40 & $0.9452 \%$ & $3.43 \mathrm{E}-03$ & $1.3783 \%$ & $3.43 \mathrm{E}-03$ & $0.8327 \%$ & $2.91 \mathrm{E}-03$ & $0.0823 \%$ & $3.29 \mathrm{E}-03$ & $0.9981 \%$ & $3.03 \mathrm{E}-03$ \\
\hline 50 & $0.8105 \%$ & $2.93 \mathrm{E}-03$ & $1.1529 \%$ & $3.52 \mathrm{E}-03$ & $0.6704 \%$ & $3.26 \mathrm{E}-03$ & $0.0572 \%$ & $3.30 \mathrm{E}-03$ & $0.7646 \%$ & $3.08 \mathrm{E}-03$ \\
\hline 60 & $0.6914 \%$ & $3.10 \mathrm{E}-03$ & $0.9408 \%$ & $3.63 \mathrm{E}-03$ & $0.5602 \%$ & $3.29 \mathrm{E}-03$ & $0.0503 \%$ & $3.46 \mathrm{E}-03$ & $0.6161 \%$ & $3.09 \mathrm{E}-03$ \\
\hline 70 & $0.5470 \%$ & $3.57 \mathrm{E}-03$ & $0.8421 \%$ & $3.75 \mathrm{E}-03$ & $0.4807 \%$ & $3.53 \mathrm{E}-03$ & $0.0376 \%$ & $3.57 \mathrm{E}-03$ & $0.5579 \%$ & $3.24 \mathrm{E}-03$ \\
\hline 80 & $0.4817 \%$ & $3.40 \mathrm{E}-03$ & $0.6799 \%$ & $4.01 \mathrm{E}-03$ & $0.4208 \%$ & $3.46 \mathrm{E}-03$ & $0.0264 \%$ & $3.68 \mathrm{E}-03$ & $0.4714 \%$ & $3.38 \mathrm{E}-03$ \\
\hline 90 & $0.4189 \%$ & $3.46 \mathrm{E}-03$ & $0.6222 \%$ & $4.34 \mathrm{E}-03$ & $0.3752 \%$ & $3.95 \mathrm{E}-03$ & $0.0234 \%$ & $4.03 \mathrm{E}-03$ & $0.4108 \%$ & $3.43 \mathrm{E}-03$ \\
\hline 100 & $0.3891 \%$ & $3.71 \mathrm{E}-03$ & $0.5891 \%$ & 4.88E-03 & $0.3376 \%$ & $4.24 \mathrm{E}-03$ & $0.0210 \%$ & 4.73E-03 & $0.3604 \%$ & $3.79 \mathrm{E}-03$ \\
\hline
\end{tabular}

Table 2. Comparing the Accelerated LR and Tian models with analytical formulae and the leading benchmark tree using the smaller step-size 

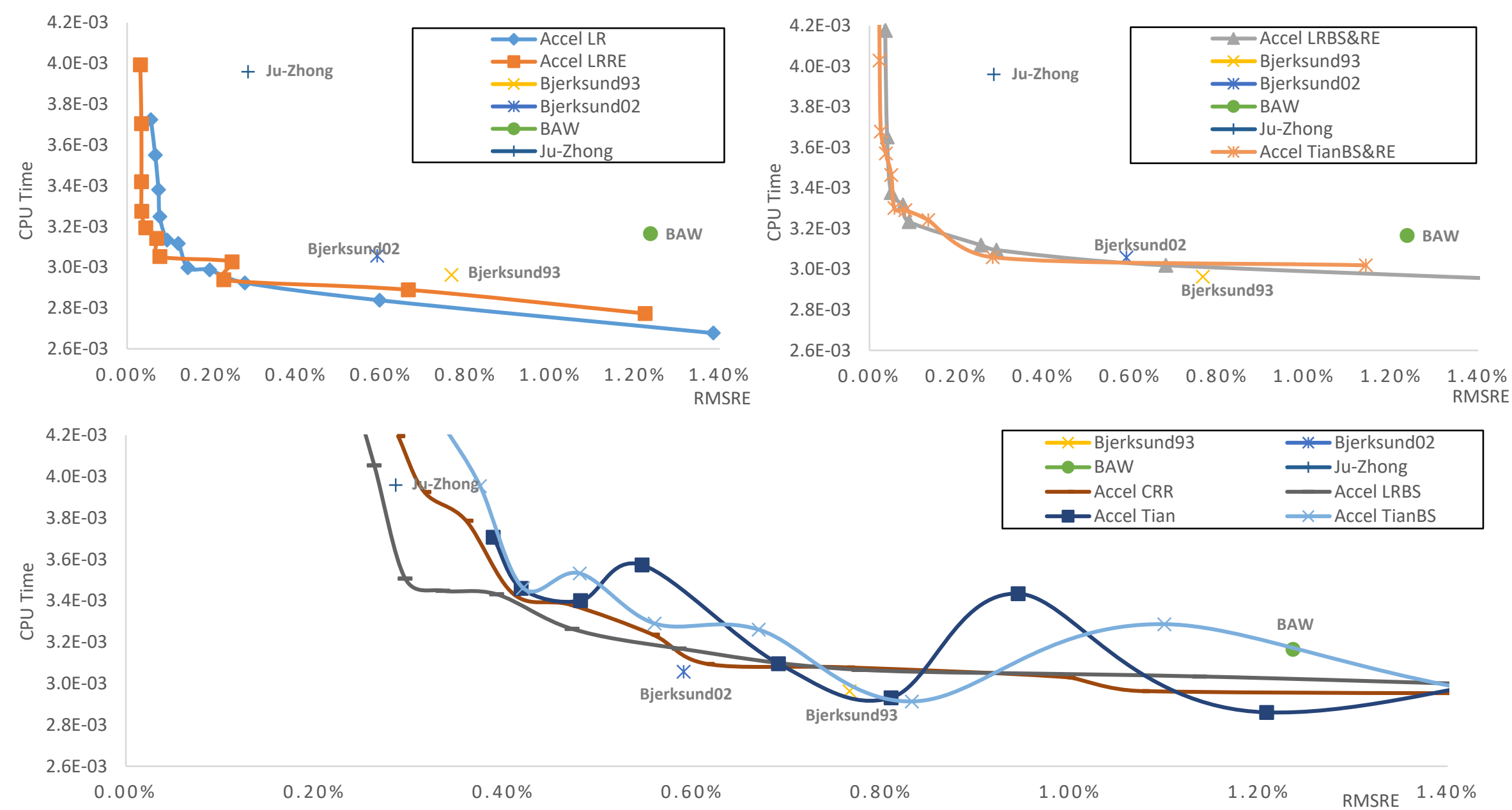

Fig. 6 Comparing Accelerated the LR and Tian models with analytical formulae and the leading benchmark tree using the smaller steps-size 


\begin{tabular}{|c|c|c|c|c|c|c|c|c|c|c|}
\hline \multirow{2}{*}{$\begin{array}{c}\text { Method } \\
\text { Steps }\end{array}$} & \multicolumn{2}{|c|}{ Accel LR } & \multicolumn{2}{|c|}{ Accel LRRE } & \multicolumn{2}{|c|}{ Accel LRBS } & \multicolumn{2}{|c|}{ Accel LRBS\&RE } & \multicolumn{2}{|c|}{ Accel CRR } \\
\hline & RMSRE & Time & RMSRE & Time & RMSRE & Time & RMSRE & Time & RMSRE & Time \\
\hline 100 & $0.0562 \%$ & $3.72 \mathrm{E}-03$ & $0.0312 \%$ & $3.99 \mathrm{E}-03$ & $0.2358 \%$ & $4.48 \mathrm{E}-03$ & $0.0327 \%$ & $5.47 \mathrm{E}-03$ & $0.3604 \%$ & $3.79 \mathrm{E}-03$ \\
\hline 200 & $0.0285 \%$ & 7.49E-03 & $0.0106 \%$ & $1.02 \mathrm{E}-02$ & $0.1173 \%$ & $1.32 \mathrm{E}-02$ & $0.0113 \%$ & $1.52 \mathrm{E}-02$ & $0.1791 \%$ & $7.80 \mathrm{E}-03$ \\
\hline 300 & $0.0193 \%$ & $1.59 \mathrm{E}-02$ & $0.0069 \%$ & $2.12 \mathrm{E}-02$ & $0.0780 \%$ & $2.76 \mathrm{E}-02$ & $0.0071 \%$ & $3.19 \mathrm{E}-02$ & $0.1079 \%$ & $1.57 \mathrm{E}-02$ \\
\hline 400 & $0.0144 \%$ & 2.67E-02 & $0.0043 \%$ & 3.37E-02 & $0.0582 \%$ & 4.37E-02 & $0.0045 \%$ & $5.47 \mathrm{E}-02$ & $0.0805 \%$ & $2.61 \mathrm{E}-02$ \\
\hline 500 & $0.0115 \%$ & 4.22E-02 & $0.0061 \%$ & $5.16 \mathrm{E}-02$ & $0.0463 \%$ & $6.78 \mathrm{E}-02$ & $0.0061 \%$ & 8.41E-02 & $0.0684 \%$ & $3.98 \mathrm{E}-02$ \\
\hline 600 & $0.0096 \%$ & $5.69 \mathrm{E}-02$ & $0.0039 \%$ & $7.38 \mathrm{E}-02$ & $0.0385 \%$ & $9.92 \mathrm{E}-02$ & $0.0040 \%$ & $1.22 \mathrm{E}-01$ & $0.0570 \%$ & $5.84 \mathrm{E}-02$ \\
\hline 700 & $0.0082 \%$ & $7.88 \mathrm{E}-02$ & $0.0030 \%$ & $9.85 \mathrm{E}-02$ & $0.0328 \%$ & $1.35 \mathrm{E}-01$ & $0.0031 \%$ & $1.63 \mathrm{E}-01$ & $0.0463 \%$ & $7.86 \mathrm{E}-02$ \\
\hline 800 & $0.0072 \%$ & $9.86 \mathrm{E}-02$ & $0.0033 \%$ & $1.26 \mathrm{E}-01$ & $0.0287 \%$ & $1.69 \mathrm{E}-01$ & $0.0034 \%$ & $2.08 \mathrm{E}-01$ & $0.0410 \%$ & $9.94 \mathrm{E}-02$ \\
\hline 900 & $0.0065 \%$ & $1.25 \mathrm{E}-01$ & $0.0035 \%$ & $1.56 \mathrm{E}-01$ & $0.0255 \%$ & $2.14 \mathrm{E}-01$ & $0.0035 \%$ & $2.68 \mathrm{E}-01$ & $0.0367 \%$ & $1.22 \mathrm{E}-01$ \\
\hline 1000 & $0.0059 \%$ & $1.71 \mathrm{E}-01$ & $0.0036 \%$ & $1.90 \mathrm{E}-01$ & $0.0229 \%$ & $2.66 \mathrm{E}-01$ & $0.0036 \%$ & $3.25 \mathrm{E}-01$ & $0.0385 \%$ & $1.53 \mathrm{E}-01$ \\
\hline Method & \multicolumn{2}{|c|}{ Accel Tian } & \multicolumn{2}{|c|}{ Accel TianRE } & \multicolumn{2}{|c|}{ Accel TianBS } & \multicolumn{4}{|c|}{ Accel TianBS\&RE } \\
\hline Steps & RMSRE & Time & RMSRE & Time & RMSRE & Time & \multicolumn{2}{|c|}{ RMSRE } & \multicolumn{2}{|c|}{ Time } \\
\hline 100 & $0.3891 \%$ & $3.71 \mathrm{E}-03$ & $0.5891 \%$ & $4.56 \mathrm{E}-03$ & $0.3376 \%$ & $4.24 \mathrm{E}-03$ & \multicolumn{2}{|c|}{$0.0210 \%$} & \multicolumn{2}{|c|}{ 4.73E-03 } \\
\hline 200 & $0.1872 \%$ & $1.09 \mathrm{E}-02$ & $0.2852 \%$ & $1.36 \mathrm{E}-02$ & $0.1688 \%$ & $1.22 \mathrm{E}-02$ & \multicolumn{2}{|c|}{$0.0082 \%$} & \multicolumn{2}{|c|}{$1.50 \mathrm{E}-02$} \\
\hline 300 & $0.1276 \%$ & $2.09 \mathrm{E}-02$ & $0.1894 \%$ & $2.59 \mathrm{E}-02$ & $0.1121 \%$ & $2.62 \mathrm{E}-02$ & \multicolumn{2}{|c|}{$0.0064 \%$} & \multicolumn{2}{|c|}{$3.33 \mathrm{E}-02$} \\
\hline 400 & $0.0954 \%$ & $3.49 \mathrm{E}-02$ & $0.1402 \%$ & $4.45 \mathrm{E}-02$ & $0.0838 \%$ & $4.66 \mathrm{E}-02$ & \multicolumn{2}{|c|}{$0.0041 \%$} & \multicolumn{2}{|c|}{$5.65 \mathrm{E}-02$} \\
\hline 500 & $0.0741 \%$ & $5.11 \mathrm{E}-02$ & $0.1155 \%$ & $6.57 \mathrm{E}-02$ & $0.0668 \%$ & $6.72 \mathrm{E}-02$ & \multicolumn{2}{|c|}{$0.0037 \%$} & \multicolumn{2}{|c|}{$8.72 \mathrm{E}-02$} \\
\hline 600 & $0.0636 \%$ & 7.44E-02 & $0.0920 \%$ & $9.18 \mathrm{E}-02$ & $0.0553 \%$ & $9.52 \mathrm{E}-02$ & \multicolumn{2}{|c|}{$0.0035 \%$} & \multicolumn{2}{|c|}{$1.22 \mathrm{E}-01$} \\
\hline 700 & $0.0557 \%$ & $9.67 \mathrm{E}-02$ & $0.0825 \%$ & $1.31 \mathrm{E}-01$ & $0.0474 \%$ & $1.29 \mathrm{E}-01$ & \multicolumn{2}{|c|}{$0.0035 \%$} & \multicolumn{2}{|c|}{$1.60 \mathrm{E}-01$} \\
\hline 800 & $0.0455 \%$ & $1.25 \mathrm{E}-01$ & $0.0693 \%$ & $1.68 \mathrm{E}-01$ & $0.0413 \%$ & $1.65 \mathrm{E}-01$ & \multicolumn{2}{|c|}{$0.0029 \%$} & \multicolumn{2}{|c|}{$2.10 \mathrm{E}-01$} \\
\hline 900 & $0.0451 \%$ & $1.57 \mathrm{E}-01$ & $0.0640 \%$ & $2.20 \mathrm{E}-01$ & $0.0363 \%$ & $2.07 \mathrm{E}-01$ & \multicolumn{2}{|c|}{$0.0028 \%$} & \multicolumn{2}{|c|}{$2.70 \mathrm{E}-01$} \\
\hline 1000 & $0.0412 \%$ & $1.91 \mathrm{E}-01$ & $0.0579 \%$ & $2.57 \mathrm{E}-01$ & $0.0327 \%$ & $2.61 \mathrm{E}-01$ & \multicolumn{2}{|c|}{$0.0026 \%$} & \multicolumn{2}{|c|}{$3.22 \mathrm{E}-01$} \\
\hline
\end{tabular}

Table 3. Comparing Accelerated LR and Tian models with the leading benchmark tree using the larger steps-size 


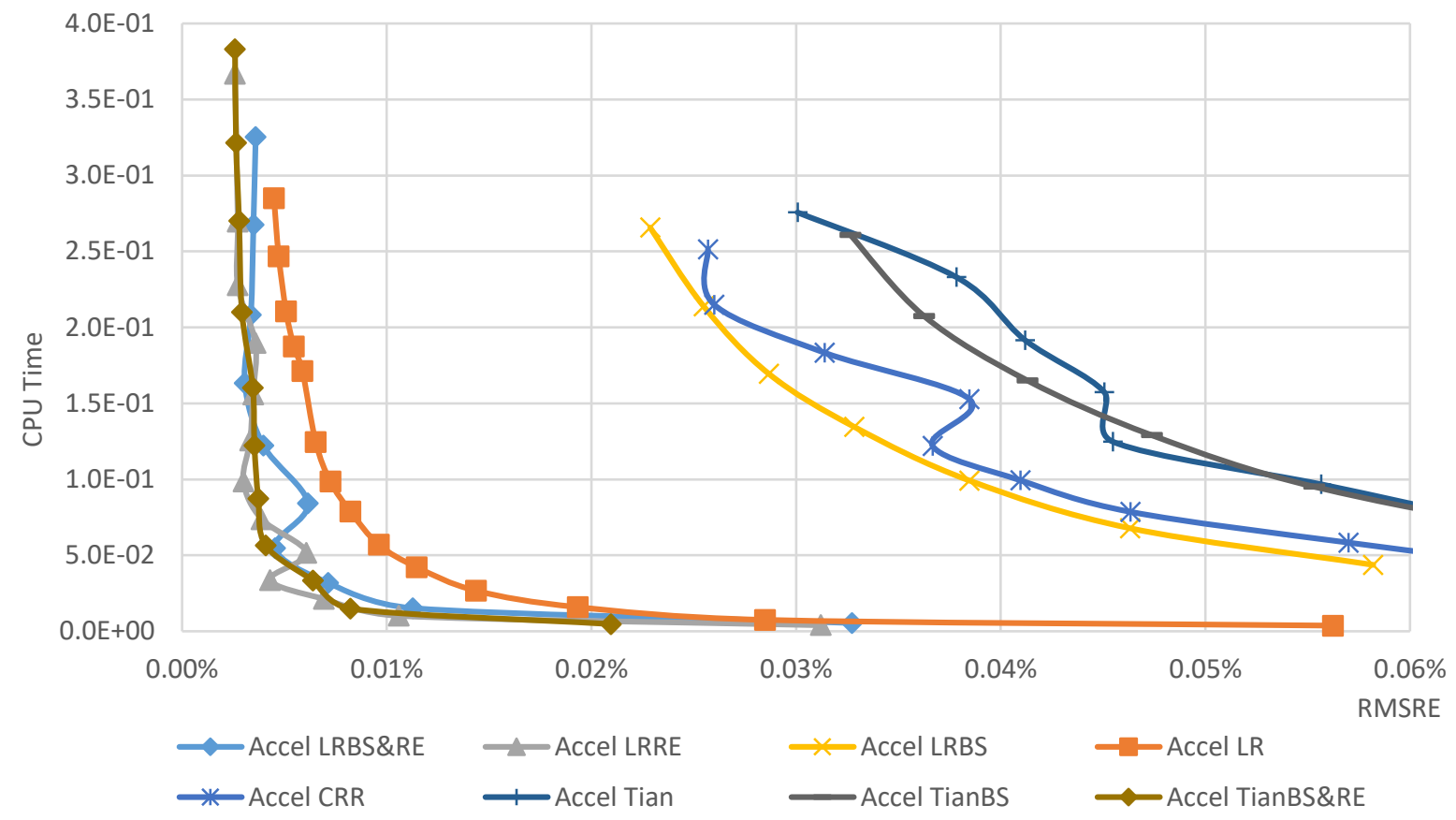

Fig. 7 Comparing Accelerated LR and Tian models with the leading benchmark tree using the larger step-size

Consistent with Pressacoo, et al. (2008), we further divide American options samples into four different groups according to their AER ratio and the moneyness classifications. We initially test the performance for each model for varying classification. First, according to the moneyness of the options, 2,330 previously used American options are divided into 1,190 in-the-money and 1,140 out-of-the-money options. Next, we separate the options according to the AER: quantitatively measured by the Pressacoo, et al. (2008) ratio of 1.4 where American value ${ }^{18}$ exceeds the European twin. The 2,330 option sample filters into 2,281 Weak options where AER $<1.40$ and 49 Strong options where the AER $\geq 1.40$. In aggregate that leaves 2,330 options being separated into 1,157 Weak-In options, 1,124 Weak-Out options, 33 Strong-In options and 16 Strong-Out options. The Weak-In and Weak-Out options are large enough to make reliable tests on while the Strong-In and Strong-Out options are not plausibly sufficient. We therefore continue to randomly generate American options, following the same criterion of parameter selection as we generated 2,330 before. We retain only Strong options up to 1,000 from the new generated options and add them to the 49 Strong options already obtained. Finally, we obtain 569 Strong-In options and 302 Strong-Out options.

Based on the results of the previous tests, Accel LR, Accel LRRE, Accel LRBS\&RE and Accel TianBS\&RE have outstanding performance in pricing American options with both lower and higher level of accuracy (small and large step-size). Here we apply these four lattice models as well as four analytical formulae to value 589 Strong-In, 302 Strong-Out, 1,157 Weak-In, and 1,124 Weak-Out

${ }^{18}$ The American value of options used here is the quick reliable estimation. 
options previously generated. For lattice models, in addition, the number of steps is ranged from 10 to 1,000. ${ }^{19}$ The corresponding RMSRE and CPU Time are reported in Table 4 and visualized in Fig. 8 and Fig. 9. First of all, we compare the difficulty of pricing different types of options. As it is shown in Table 4, the RMSRE generated by lattice models for pricing Weak options is much less than when typically pricing Strong options, at different numbers of steps. This indicates that, in general, pricing Strong options is more difficult than Weak options for lattice models. For analytical formulae, however, Strong-Out options are much harder to value than Weak-Out options while Strong-In options are easier to price than Weak-In options. Secondly, we compare analytical formulae and lattice models with a smaller number of steps $(\leq 100)$ in pricing different types of options. To make the observation clearer, only Accel LR and Accel LRRE, the two top performing lattice models, are presented in Fig. 8. When pricing Weak (In/Out) options, lattice models easily surpass analytical formulae since the curves are clearly closer to the origin relative to the four analytical formulae points. More specifically, Accel LRRE offers the best performance in pricing Weak options when considering both efficiency and stability. On the contrary, for pricing Strong (In/Out) options, lattice models have no significant advantage over analytical formulae in achieving the same level of accuracy. ${ }^{20}$ The four analytical formulae points are in the vicinity of the lattice models curves. However, we should not overlook the natural advantage of lattice models in offering granular combinations of speed and accuracy. In particular, the Accel LR provides the best performance in valuing Strong options. Thirdly, we consider four lattice models in terms of valuing varying types of options with a larger number of steps $(>100)$ in Fig. 9. The Accel TianBS\&RE clearly performs best in pricing Strong-In and Strong-Out and offers relatively better performance in valuing Weak-In options. For Weak-Out options, Accel LRBS\&RE is associated with the best performance by virtue that Accel TianBS\&RE produces a lower level of efficiency and Accel LRRE lacks smooth convergence. In practice, we can determine the moneyness and the American quality of options (Strong/Weak/In/Out) and then select the appropriate lattice model to for estimation.

\footnotetext{
${ }^{19}$ For pricing Strong-In options, the steps are ranged from 20 to 1,000. In addition, the accuracy and speed level of Accel LR for pricing Strong-In options improves more slowly than the other three lattice models as the number of steps increasing. To make the results comparable, we broaden the step range of Accel LR from 20 to 3,000.

${ }^{20}$ This only applies in a narrow minority of cases by virtue that strong options are quite rare.
} 
a) Strong out of the Money Sample

\begin{tabular}{|c|c|c|c|c|c|c|c|c|c|c|}
\hline \multirow{3}{*}{$\begin{array}{c}\text { Method } \\
\text { Steps } \\
10\end{array}$} & \multicolumn{2}{|c|}{ Accel LR } & \multicolumn{2}{|c|}{ Accel LRRE } & \multicolumn{2}{|c|}{ Accel LRBS\&RE } & \multicolumn{2}{|c|}{ Accel TianBS\&RE } & & \\
\hline & RMSRE & Time & RMSRE & Time & RMSRE & Time & RMSRE & Time & \multicolumn{2}{|c|}{ BAW } \\
\hline & $3.0471 \%$ & $2.43 \mathrm{E}-03$ & $4.7669 \%$ & $3.37 \mathrm{E}-03$ & $4.7800 \%$ & $3.69 \mathrm{E}-03$ & $2.8573 \%$ & $3.31 \mathrm{E}-03$ & RMSRE & Time \\
\hline 20 & $1.5064 \%$ & $3.26 \mathrm{E}-03$ & $1.7461 \%$ & $3.32 \mathrm{E}-03$ & $1.7593 \%$ & $3.36 \mathrm{E}-03$ & $0.9883 \%$ & $3.30 \mathrm{E}-03$ & $3.1896 \%$ & $3.67 \mathrm{E}-03$ \\
\hline 30 & $0.9760 \%$ & $3.33 \mathrm{E}-03$ & $0.8530 \%$ & $3.77 \mathrm{E}-03$ & $0.9019 \%$ & $3.57 \mathrm{E}-03$ & $0.5264 \%$ & $3.59 \mathrm{E}-03$ & & \\
\hline 50 & $0.5733 \%$ & $3.81 \mathrm{E}-03$ & $0.3894 \%$ & $3.61 \mathrm{E}-03$ & $0.4090 \%$ & $3.47 \mathrm{E}-03$ & $0.4090 \%$ & 3.84E-03 & \multicolumn{2}{|c|}{ Bjerksund93 } \\
\hline 100 & $0.2733 \%$ & $3.75 \mathrm{E}-03$ & $0.1655 \%$ & $3.97 \mathrm{E}-03$ & $0.1674 \%$ & $4.83 \mathrm{E}-03$ & $0.0788 \%$ & 5.38E-03 & RMSRE & Time \\
\hline 200 & $0.1339 \%$ & $7.63 \mathrm{E}-03$ & $0.0915 \%$ & $9.35 \mathrm{E}-03$ & $0.0924 \%$ & $1.36 \mathrm{E}-02$ & $0.0380 \%$ & $1.79 \mathrm{E}-02$ & $1.6936 \%$ & $3.33 \mathrm{E}-03$ \\
\hline 300 & $0.0864 \%$ & $1.55 \mathrm{E}-02$ & $0.0492 \%$ & $1.75 \mathrm{E}-02$ & $0.0502 \%$ & $2.68 \mathrm{E}-02$ & $0.0186 \%$ & $3.63 \mathrm{E}-02$ & & \\
\hline 400 & $0.0638 \%$ & $2.35 \mathrm{E}-02$ & $0.0326 \%$ & $2.88 \mathrm{E}-02$ & $0.0334 \%$ & $5.47 \mathrm{E}-02$ & $0.0153 \%$ & $5.64 \mathrm{E}-02$ & \multicolumn{2}{|c|}{ Bjerksund02 } \\
\hline 500 & $0.0508 \%$ & $3.57 \mathrm{E}-02$ & $0.0193 \%$ & 4.41E-02 & $0.0199 \%$ & 7.43E-02 & $0.0105 \%$ & 8.17E-02 & RMSRE & Time \\
\hline 600 & $0.0414 \%$ & $4.50 \mathrm{E}-02$ & $0.0185 \%$ & $6.00 \mathrm{E}-02$ & $0.0188 \%$ & $1.05 \mathrm{E}-01$ & $0.0081 \%$ & $1.12 \mathrm{E}-01$ & $1.6980 \%$ & $3.47 \mathrm{E}-03$ \\
\hline 700 & $0.0343 \%$ & $6.22 \mathrm{E}-02$ & $0.0172 \%$ & 7.97E-02 & $0.0188 \%$ & $1.12 \mathrm{E}-01$ & $0.0086 \%$ & $1.55 \mathrm{E}-01$ & & \\
\hline 800 & $0.0294 \%$ & 8.77E-02 & $0.0128 \%$ & $1.02 \mathrm{E}-01$ & $0.0131 \%$ & $1.91 \mathrm{E}-01$ & $0.0062 \%$ & $2.09 \mathrm{E}-01$ & \multicolumn{2}{|c|}{ JuZhong } \\
\hline 900 & $0.0265 \%$ & $9.89 \mathrm{E}-02$ & $0.0091 \%$ & $1.23 \mathrm{E}-01$ & $0.0094 \%$ & $2.28 \mathrm{E}-01$ & $0.0065 \%$ & $2.71 \mathrm{E}-01$ & RMSRE & Time \\
\hline 1000 & $0.0230 \%$ & $1.18 \mathrm{E}-01$ & $0.0109 \%$ & $1.50 \mathrm{E}-01$ & $0.0112 \%$ & $2.77 \mathrm{E}-01$ & $0.0055 \%$ & 2.99E-01 & $2.0285 \%$ & $3.29 \mathrm{E}-03$ \\
\hline
\end{tabular}

b) Strong in the Money Sample

\begin{tabular}{|c|c|c|c|c|c|c|c|c|c|c|c|}
\hline \multirow{3}{*}{$\begin{array}{c}\text { Method } \\
\text { Steps } \\
20\end{array}$} & \multicolumn{2}{|c|}{ Accel LRRE } & \multicolumn{2}{|c|}{ Accel LRBS\&RE } & \multicolumn{2}{|c|}{ Accel TianBS\&RE } & \multirow[b]{2}{*}{ Steps } & \multicolumn{2}{|c|}{ Accel LR } & & \\
\hline & RMSRE & Time & RMSRE & Time & RMSRE & Time & & RMSRE & Time & \multicolumn{2}{|c|}{ BAW } \\
\hline & $0.9092 \%$ & $3.53 \mathrm{E}-03$ & $0.9145 \%$ & $3.50 \mathrm{E}-03$ & $0.6363 \%$ & $3.48 \mathrm{E}-03$ & 20 & $0.8893 \%$ & $2.99 \mathrm{E}-03$ & RMSRE & Time \\
\hline 30 & $0.6415 \%$ & $3.42 \mathrm{E}-03$ & $0.6421 \%$ & $3.46 \mathrm{E}-03$ & $0.3932 \%$ & $3.64 \mathrm{E}-03$ & 30 & $0.5916 \%$ & $3.22 \mathrm{E}-03$ & $0.5517 \%$ & $3.35 \mathrm{E}-03$ \\
\hline 50 & $0.2822 \%$ & $3.55 \mathrm{E}-03$ & $0.2841 \%$ & $3.82 \mathrm{E}-03$ & $0.1869 \%$ & $3.51 \mathrm{E}-03$ & 50 & $0.3657 \%$ & $3.28 \mathrm{E}-03$ & & \\
\hline 100 & $0.1722 \%$ & $3.58 \mathrm{E}-03$ & $0.1724 \%$ & $5.06 \mathrm{E}-03$ & $0.1043 \%$ & 4.94E-03 & 100 & $0.1851 \%$ & $3.32 \mathrm{E}-03$ & Bjer & ind93 \\
\hline 150 & $0.0994 \%$ & $5.84 \mathrm{E}-03$ & $0.0995 \%$ & $9.98 \mathrm{E}-03$ & $0.0677 \%$ & $1.03 \mathrm{E}-02$ & 150 & $0.1220 \%$ & $4.31 \mathrm{E}-03$ & RMSRE & Time \\
\hline 200 & $0.0825 \%$ & $9.51 \mathrm{E}-03$ & $0.0826 \%$ & $1.56 \mathrm{E}-02$ & $0.0438 \%$ & $1.44 \mathrm{E}-02$ & 200 & $0.0921 \%$ & $7.46 \mathrm{E}-03$ & $0.2556 \%$ & $3.27 \mathrm{E}-03$ \\
\hline 300 & $0.0516 \%$ & $1.69 \mathrm{E}-02$ & $0.0515 \%$ & $3.17 \mathrm{E}-02$ & $0.0339 \%$ & $3.13 \mathrm{E}-02$ & 300 & $0.0647 \%$ & $1.34 \mathrm{E}-02$ & & \\
\hline 400 & $0.0364 \%$ & $2.79 \mathrm{E}-02$ & $0.0364 \%$ & $5.45 \mathrm{E}-02$ & $0.0225 \%$ & $5.02 \mathrm{E}-02$ & 500 & $0.0366 \%$ & $3.39 \mathrm{E}-02$ & Bjer & Ind02 \\
\hline 500 & $0.0297 \%$ & $5.76 \mathrm{E}-02$ & $0.0296 \%$ & $8.18 \mathrm{E}-02$ & $0.0167 \%$ & $7.33 \mathrm{E}-02$ & 800 & $0.0213 \%$ & 7.59E-02 & RMSRE & Time \\
\hline 600 & $0.0240 \%$ & $6.85 \mathrm{E}-02$ & $0.0240 \%$ & $1.07 \mathrm{E}-01$ & $0.0140 \%$ & $1.05 \mathrm{E}-01$ & 1000 & $0.0174 \%$ & $1.32 \mathrm{E}-01$ & $0.2378 \%$ & $3.45 \mathrm{E}-03$ \\
\hline 700 & $0.0173 \%$ & 8.87E-02 & $0.0173 \%$ & $1.35 \mathrm{E}-01$ & $0.0117 \%$ & $1.41 \mathrm{E}-01$ & 1500 & $0.0116 \%$ & $2.61 \mathrm{E}-01$ & & \\
\hline 800 & $0.0147 \%$ & $9.55 \mathrm{E}-02$ & $0.0148 \%$ & $1.74 \mathrm{E}-01$ & $0.0089 \%$ & $1.86 \mathrm{E}-01$ & 2000 & $0.0085 \%$ & 4.73E-01 & & ong \\
\hline 900 & $0.0125 \%$ & $1.14 \mathrm{E}-01$ & $0.0125 \%$ & $2.31 \mathrm{E}-01$ & $0.0081 \%$ & 2.34E-01 & 2500 & $0.0067 \%$ & $6.92 \mathrm{E}-01$ & RMSRE & Time \\
\hline 1000 & $0.0113 \%$ & $1.50 \mathrm{E}-01$ & $0.0113 \%$ & $2.59 \mathrm{E}-01$ & $0.0073 \%$ & $2.68 \mathrm{E}-01$ & 3000 & $0.0054 \%$ & 9.92E-01 & $0.1550 \%$ & $3.41 \mathrm{E}-03$ \\
\hline
\end{tabular}


c) Weak out of the Money Sample

\begin{tabular}{|c|c|c|c|c|c|c|c|c|c|c|}
\hline \multirow{2}{*}{$\begin{array}{c}\text { Method } \\
\text { Steps } \\
\end{array}$} & \multicolumn{2}{|c|}{ Accel LR } & \multicolumn{2}{|c|}{ Accel LRRE } & \multicolumn{2}{|c|}{ Accel LRBS\&RE } & \multicolumn{2}{|c|}{ Accel TianBS\&RE } & \multirow{2}{*}{\multicolumn{2}{|c|}{ BAW }} \\
\hline & RMSRE & Time & RMSRE & Time & RMSRE & Time & RMSRE & Time & & \\
\hline 10 & $0.4970 \%$ & $2.82 \mathrm{E}-03$ & $0.4675 \%$ & $2.90 \mathrm{E}-03$ & $0.5101 \%$ & $3.01 \mathrm{E}-03$ & $1.5608 \%$ & $3.15 \mathrm{E}-03$ & RMSRE & Time \\
\hline 20 & $0.2418 \%$ & $2.86 \mathrm{E}-03$ & $0.0998 \%$ & $2.98 \mathrm{E}-03$ & $0.2667 \%$ & $3.06 \mathrm{E}-03$ & $0.3267 \%$ & $3.51 \mathrm{E}-03$ & $1.5850 \%$ & $3.21 \mathrm{E}-03$ \\
\hline 30 & $0.1612 \%$ & $3.24 \mathrm{E}-03$ & $0.0724 \%$ & $3.15 \mathrm{E}-03$ & $0.1063 \%$ & $3.34 \mathrm{E}-03$ & $0.1509 \%$ & $3.29 \mathrm{E}-03$ & \multirow{2}{*}{\multicolumn{2}{|c|}{ Bjerksund93 }} \\
\hline 50 & $0.0971 \%$ & $3.48 \mathrm{E}-03$ & $0.0305 \%$ & $3.50 \mathrm{E}-03$ & $0.0521 \%$ & $3.55 \mathrm{E}-03$ & $0.0609 \%$ & $3.63 \mathrm{E}-03$ & & \\
\hline 100 & $0.0493 \%$ & $4.43 \mathrm{E}-03$ & $0.0077 \%$ & 5.03E-03 & $0.0157 \%$ & $5.76 \mathrm{E}-03$ & $0.0188 \%$ & $6.57 \mathrm{E}-03$ & RMSRE & Time \\
\hline 200 & $0.0252 \%$ & $1.05 \mathrm{E}-02$ & $0.0033 \%$ & $1.26 \mathrm{E}-02$ & $0.0061 \%$ & $1.78 \mathrm{E}-02$ & $0.0075 \%$ & $1.85 \mathrm{E}-02$ & $0.7797 \%$ & $3.49 \mathrm{E}-03$ \\
\hline 300 & $0.0168 \%$ & $1.95 \mathrm{E}-02$ & $0.0034 \%$ & $2.35 \mathrm{E}-02$ & $0.0041 \%$ & $3.48 \mathrm{E}-02$ & $0.0049 \%$ & $3.64 \mathrm{E}-02$ & & \\
\hline 400 & $0.0128 \%$ & $3.08 \mathrm{E}-02$ & $0.0034 \%$ & $3.80 \mathrm{E}-02$ & $0.0039 \%$ & $6.13 \mathrm{E}-02$ & $0.0046 \%$ & $6.41 \mathrm{E}-02$ & \multicolumn{2}{|c|}{ Bjerksund02 } \\
\hline 500 & $0.0104 \%$ & $4.80 \mathrm{E}-02$ & $0.0034 \%$ & $5.58 \mathrm{E}-02$ & $0.0036 \%$ & 8.53E-02 & $0.0042 \%$ & $9.07 \mathrm{E}-02$ & RMSRE & Time \\
\hline 600 & $0.0088 \%$ & $6.61 \mathrm{E}-02$ & $0.0034 \%$ & 8.35E-02 & $0.0035 \%$ & $1.24 \mathrm{E}-01$ & $0.0038 \%$ & $1.33 \mathrm{E}-01$ & $0.4372 \%$ & $3.39 \mathrm{E}-03$ \\
\hline 700 & $0.0077 \%$ & 8.29E-02 & $0.0034 \%$ & $1.05 \mathrm{E}-01$ & $0.0035 \%$ & $1.60 \mathrm{E}-01$ & $0.0036 \%$ & $1.82 \mathrm{E}-01$ & & \\
\hline 800 & $0.0069 \%$ & $1.13 \mathrm{E}-01$ & $0.0034 \%$ & $1.41 \mathrm{E}-01$ & $0.0034 \%$ & 2.09E-01 & $0.0037 \%$ & 2.29E-01 & \multicolumn{2}{|c|}{ JuZhong } \\
\hline 900 & $0.0063 \%$ & $1.38 \mathrm{E}-01$ & $0.0034 \%$ & $1.75 \mathrm{E}-01$ & $0.0034 \%$ & $2.61 \mathrm{E}-01$ & $0.0035 \%$ & $2.91 \mathrm{E}-01$ & RMSRE & Time \\
\hline 1000 & $0.0059 \%$ & $1.69 \mathrm{E}-01$ & $0.0034 \%$ & $1.98 \mathrm{E}-01$ & $0.0034 \%$ & $3.22 \mathrm{E}-01$ & $0.0035 \%$ & $3.38 \mathrm{E}-01$ & $0.2939 \%$ & $3.89 \mathrm{E}-03$ \\
\hline \multicolumn{11}{|c|}{ d) Weak in the Money Sample } \\
\hline Method & \multicolumn{2}{|c|}{ Accel LR } & \multicolumn{2}{|c|}{ Accel LRRE } & \multicolumn{2}{|c|}{ Accel LRBS\&RE } & \multicolumn{2}{|c|}{ Accel TianBS\&RE } & \multirow{2}{*}{\multicolumn{2}{|c|}{ BAW }} \\
\hline Steps & RMSRE & Time & RMSRE & Time & RMSRE & Time & RMSRE & Time & & \\
\hline 10 & $0.4463 \%$ & $2.67 \mathrm{E}-03$ & $0.3229 \%$ & $2.78 \mathrm{E}-03$ & $0.3143 \%$ & $3.22 \mathrm{E}-03$ & $0.2451 \%$ & $3.22 \mathrm{E}-03$ & RMSRE & Time \\
\hline 20 & $0.2249 \%$ & 2.73E-03 & $0.0747 \%$ & $2.86 \mathrm{E}-03$ & $0.0943 \%$ & $3.44 \mathrm{E}-03$ & $0.1007 \%$ & $3.33 \mathrm{E}-03$ & $0.6010 \%$ & $3.43 \mathrm{E}-03$ \\
\hline 30 & $0.1543 \%$ & $3.41 \mathrm{E}-03$ & $0.0549 \%$ & $3.46 \mathrm{E}-03$ & $0.0598 \%$ & 3.47E-03 & $0.1007 \%$ & $3.56 \mathrm{E}-03$ & & \\
\hline 50 & $0.0931 \%$ & $3.51 \mathrm{E}-03$ & $0.0409 \%$ & $3.35 \mathrm{E}-03$ & $0.0421 \%$ & $3.69 \mathrm{E}-03$ & $0.0248 \%$ & $3.71 \mathrm{E}-03$ & Bjer & ad93 \\
\hline 100 & $0.0458 \%$ & $3.85 \mathrm{E}-03$ & $0.0150 \%$ & 4.77E-03 & $0.0151 \%$ & $5.41 \mathrm{E}-03$ & $0.0109 \%$ & 5.39E-03 & RMSRE & Time \\
\hline 200 & $0.0225 \%$ & $9.49 \mathrm{E}-03$ & $0.0069 \%$ & $1.23 \mathrm{E}-02$ & $0.0069 \%$ & $1.75 \mathrm{E}-02$ & $0.0050 \%$ & $1.89 \mathrm{E}-02$ & $0.4111 \%$ & $3.38 \mathrm{E}-03$ \\
\hline 300 & $0.0149 \%$ & $1.95 \mathrm{E}-02$ & $0.0035 \%$ & 2.39E-02 & $0.0035 \%$ & 3.64E-02 & $0.0026 \%$ & $3.84 \mathrm{E}-02$ & & \\
\hline 400 & $0.0113 \%$ & $3.06 \mathrm{E}-02$ & $0.0030 \%$ & $3.76 \mathrm{E}-02$ & $0.0030 \%$ & $5.65 \mathrm{E}-02$ & $0.0019 \%$ & $6.54 \mathrm{E}-02$ & Bjer & $\mathrm{d} 02$ \\
\hline 500 & $0.0088 \%$ & 4.33E-02 & $0.0019 \%$ & $5.41 \mathrm{E}-02$ & $0.0019 \%$ & 8.89E-02 & $0.0015 \%$ & $9.80 \mathrm{E}-02$ & RMSRE & Time \\
\hline 600 & $0.0076 \%$ & $6.06 \mathrm{E}-02$ & $0.0019 \%$ & 7.49E-02 & $0.0019 \%$ & $1.18 \mathrm{E}-01$ & $0.0015 \%$ & $1.37 \mathrm{E}-01$ & $0.3213 \%$ & $3.23 \mathrm{E}-03$ \\
\hline 700 & $0.0065 \%$ & 7.79E-02 & $0.0014 \%$ & 9.89E-02 & $0.0014 \%$ & $1.60 \mathrm{E}-01$ & $0.0011 \%$ & $1.94 \mathrm{E}-01$ & & \\
\hline 800 & $0.0056 \%$ & $1.04 \mathrm{E}-01$ & $0.0012 \%$ & $1.33 \mathrm{E}-01$ & $0.0012 \%$ & $2.06 \mathrm{E}-01$ & $0.0009 \%$ & $2.56 \mathrm{E}-01$ & & \\
\hline 900 & $0.0050 \%$ & $1.33 \mathrm{E}-01$ & $0.0013 \%$ & $1.58 \mathrm{E}-01$ & $0.0012 \%$ & $2.65 \mathrm{E}-01$ & $0.0009 \%$ & $2.70 \mathrm{E}-01$ & RMSRE & Time \\
\hline 1000 & $0.0046 \%$ & $1.53 \mathrm{E}-01$ & $0.0011 \%$ & $1.96 \mathrm{E}-01$ & $0.0010 \%$ & 3.32E-01 & $0.0007 \%$ & $3.17 \mathrm{E}-01$ & $0.1299 \%$ & $3.97 \mathrm{E}-03$ \\
\hline
\end{tabular}

Table. 4 Comparing Accelerated LR and Tian models with analytical formulae for pricing strong/weak in/out-of the money options 

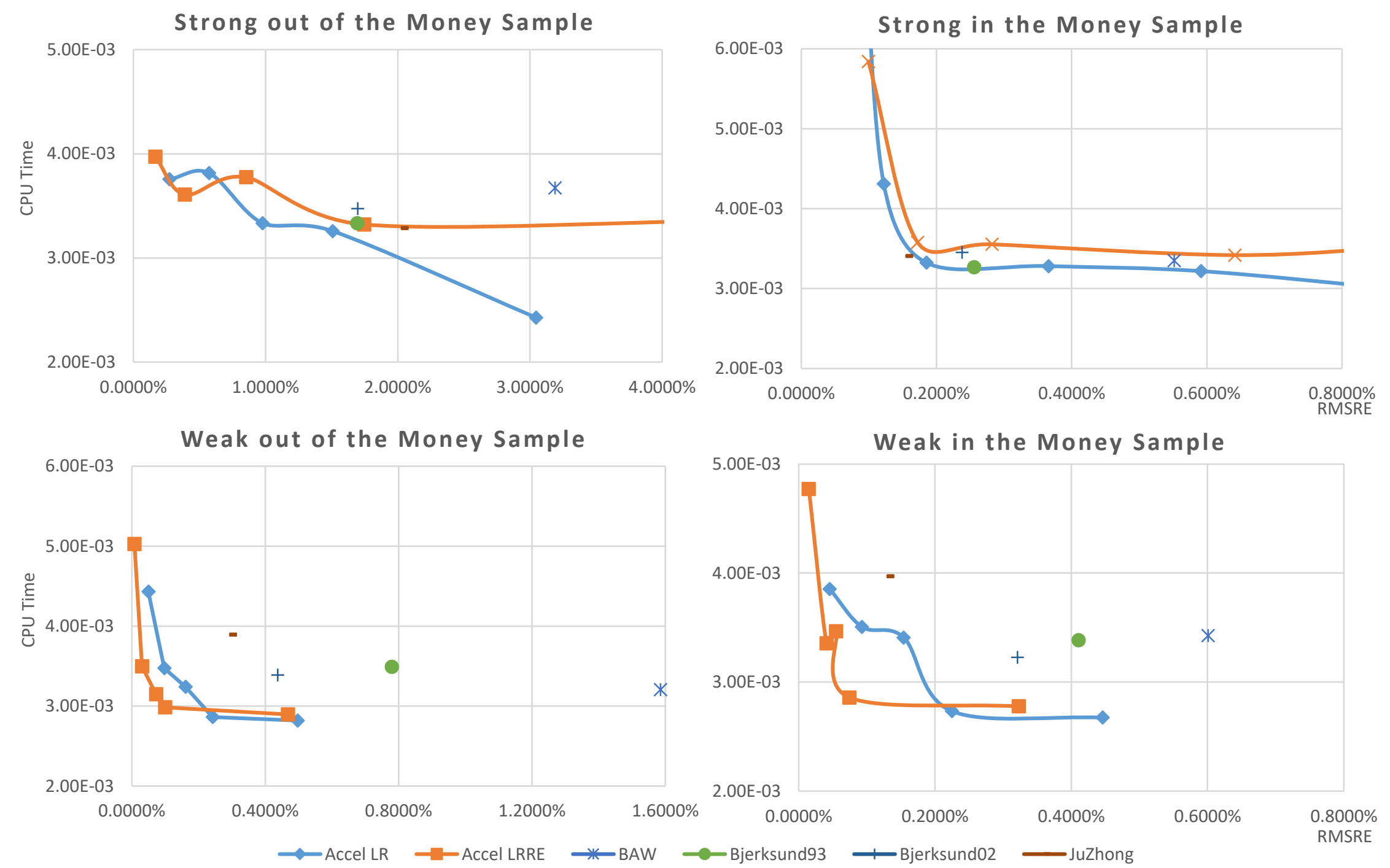

Fig. 8 Comparing optimal lattice models with analytical formulae for pricing strong/weak in/out-of the money options with smaller step size ( $\leq 100)$ 
Strong out of the Money Sample

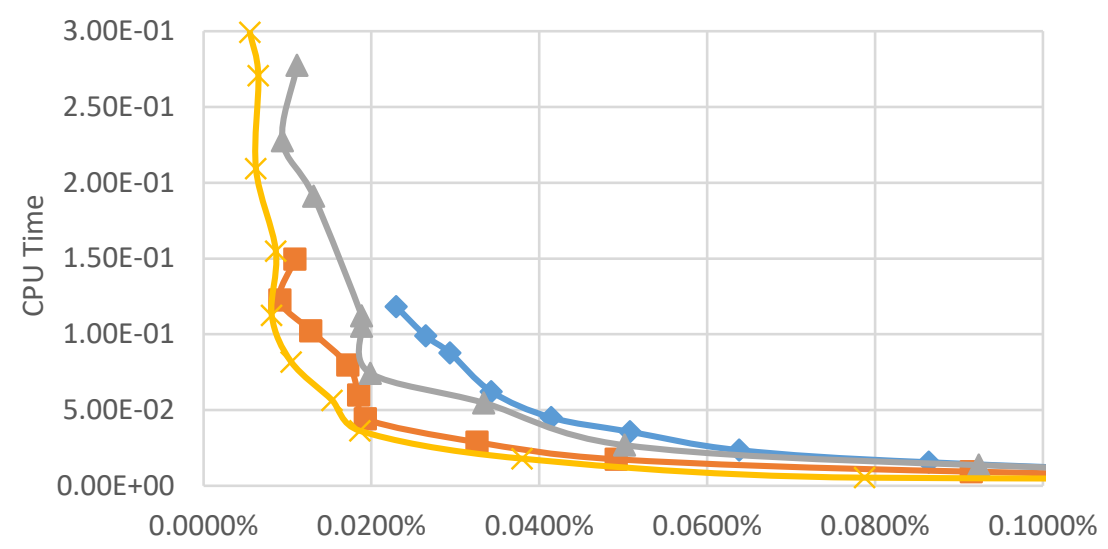

Weak out of the Money Sample

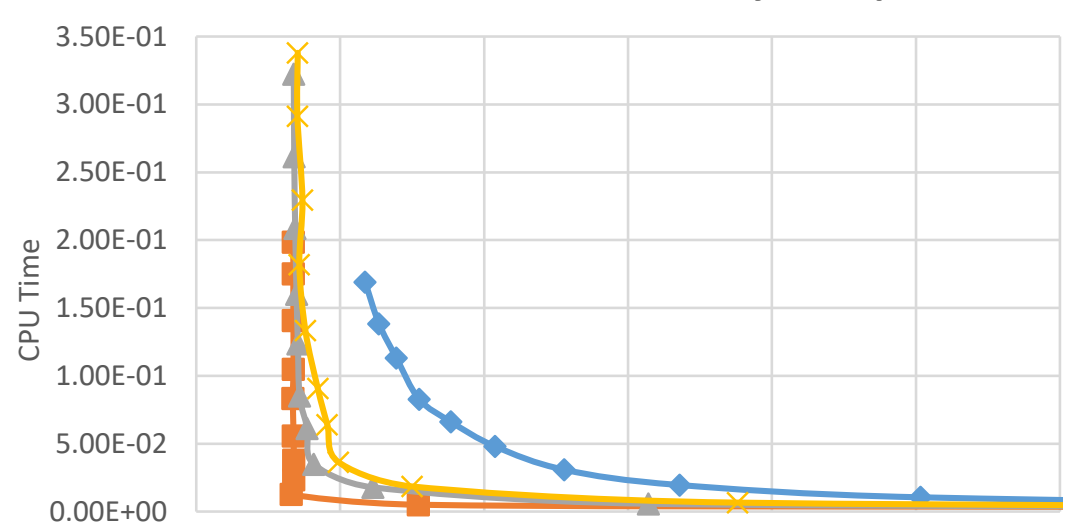

$0.0000 \% 0.0050 \% 0.0100 \% 0.0150 \% 0.0200 \% 0.0250 \% 0.0300 \%$

\section{Strong in the Money Sample}

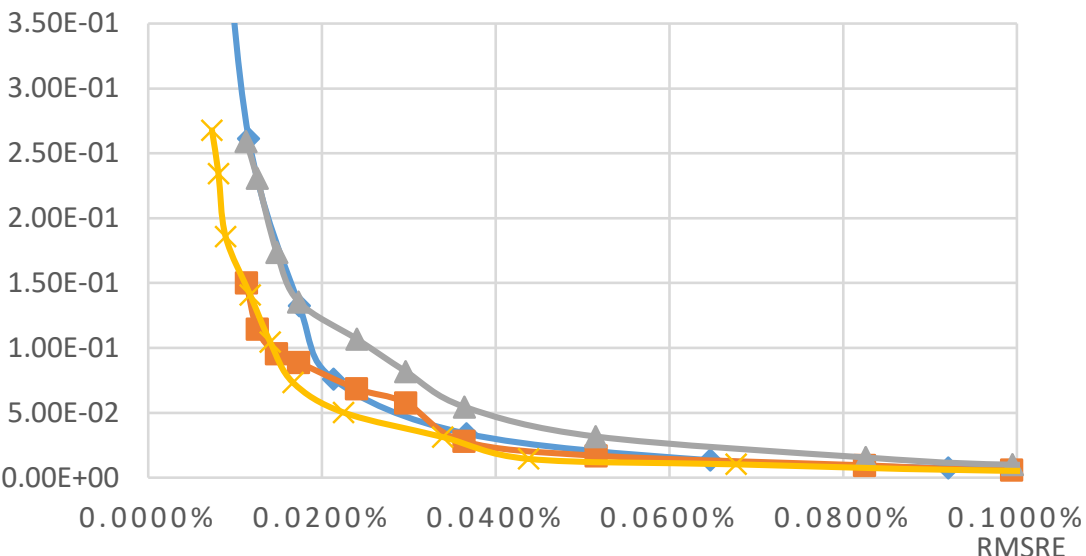

Weak in the Money Sample

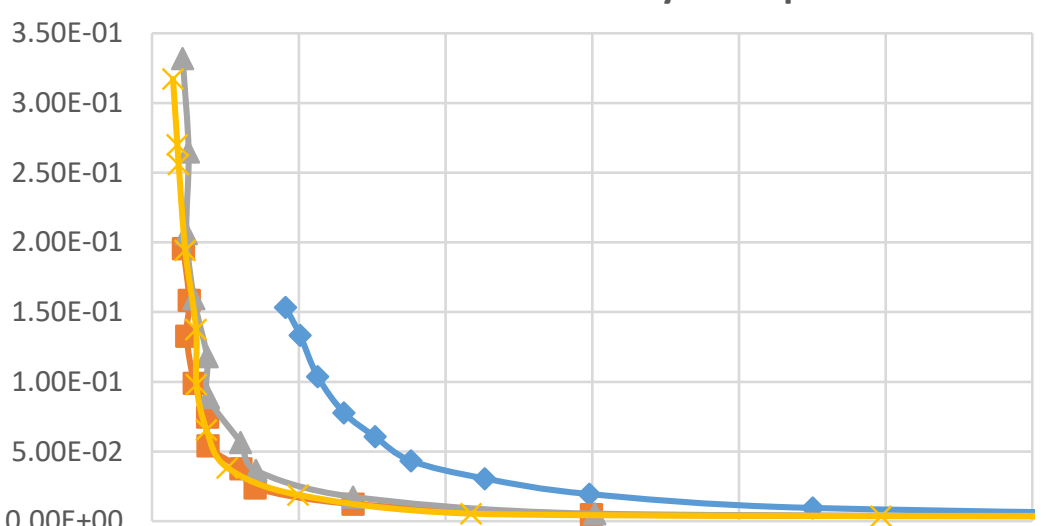

$0.0000 \% 0.0050 \% 0.0100 \% 0.0150 \% 0.0200 \% 0.0250 \% 0.0300 \%$ RMSRE $\multimap$ Accel LR $\multimap$ Accel LRRE $\multimap$ Accel LRBS\&RE $\multimap$ Accel TianBS\&RE

Fig. 9 Comparing optimal lattice models for pricing strong/weak in/out-of the money options with larger step size (> 100) 


\section{Conclusion}

In this paper, we extend the insights developed in Shang and Byrne (2019) which were originally applied uniquely to the CRR tree. We set out the conditions under which the Intelligent Lattice Search Algorithm framework can be used to promptly detect the optimal exercise boundary for a LR and Tian tree. We apply dynamic memory and truncate the LR and Tian lattice where appropriate but are careful not to disturb the original estimation. Furthermore, Black-Scholes and Richardson extrapolation modifications to the tree structures can also be applied individually and/or together to improve the accuracy of the LR and Tian models. The speed and accuracy we obtain can be made to outperform the analytical models here. This holds for all four analytical formulae that are known to have a wide dispersion of varying efficiency and error attributes. This finding reverses the received wisdom that closed form solutions do better in producing faster option values. Unlike the analytical models, the accelerated lattices however can also be made arbitrarily accurate. That is, lattices provide users with a highly granular pull-down menu choice to optimise speed relative to accuracy. One can move almost seamlessly between varying levels of precision.

Consistent with the literature, we also applied multi-dimensional efficiency tests. We benchmarked varying configurations of the LR and Tian models using several thousand random sets of parameters with each permutation linked to a given step size. This culminates with a very cleanly defined locus of accuracy relative to speed trade-offs for varying mesh densities. Our lower density meshes for Accel LR and Accel LRRE lattices prove to be more efficient than well-known analytical models prominent in the literature, achieving a higher level of accuracy. We can easily reach arbitrary levels of precision by using higher density mesh lattices. The Accel TianBS\&RE model possesses the best performance compared with other LR and Tian models and the leading benchmark tree, Accel CRR model. We further randomly generate additional options parameters and divide them into four groups according to the deepness of American quality and the moneyness of the option. We found that four efficient lattice models, including Accel LR, Accel LRRE, Accel LRBS\&RE and Accel TianBS\&RE, easily surpass analytical formulae when pricing Weak options and have comparable performance with analytical formulae in valuing Strong options. Lattices importantly afford an explicit trade-off locus between accuracy and speed that can be navigated according to predetermined precision tolerance levels and option types. In practice, with a higher tolerance for error (accepting a lower level of accuracy), Accel LRRE is recommended for pricing Weak options and Accel LR should be applied to value Strong options. To obtain a higher level of accuracy (lower tolerance for error), the Accel TianBS\&RE is recommended in pricing Strong-In, Strong-Out and Weak-In options while Accel LRBS\&RE should be used for valuing Weak-Out options. A corollary of this analysis suggests that large scale option valuation should dynamically apply varying types of lattice technique to make best use of computing resources. This should have practical relevance both in academia and trading platforms where real-time metrics like Implied Volatility are reported to traders at very high frequency. 


\section{References}

Amin, K., \& Khanna, A. (1994). Convergence of American option values from discrete-to continuoustime financial models. Mathematical Finance, 4(4), 289-304. https://doi.org/10.1111/j.1467-9965. 1994.tb00059.x.

Areal, N., \& Rodrigues, A. (2013). Fast trees for options with discrete dividends. Journal of Derivatives, 21(1), 49-63. https://doi.org/10.3905/jod.2013.21.1.049.

Barone-Adesi, G., \& Whaley, R.E. (1987). Efficient analytic approximation of American option values. Journal of Finance, 42(2), 301-320. https://doi.org/10.1111/j.1540-6261.1987.tb02569.x.

Basso, A., Nardon, M., \& Pianca, P. (2002). Discrete and continuous time approximations of the optimal exercise boundary of American options. Quaderni del Dipartimento di Matematica Applicata, 105.

Basso, A., Nardon, M., \& Pianca, P. (2004). A two-step simulation procedure to analyze the exercise features of American options. Decisions in Economics and Finance, 27(1), 35-56. https://doi.org/ 10.1007/s10203-004-0045-2.

Bjerksund, P., \& Stensland, G. (1993). Closed-form approximation of American options. Scandinavian Journal of Management, 9, 87-99. https://doi.org/10.1016/0956-5221(93)90009-H.

Bjerksund, P., \& Stensland, G. (2002). Closed form valuation of American options. Technical Report. Norwegian School of Economics and Business Administration, Department of Finance and Management Science. https://doi.org/10.1016/0956-5221(93)90009-H.

Black, F., \& Scholes, M. (1973). The pricing of options and corporate liabilities. Journal of Political Economy, 81(3), 637-654. https://doi.org/10.1086/260062.

Boyle, P. P. (1986). Option valuation using a three-jump process. International Options Journal, 3, 712.

Brennan, M.J., \& Schwartz, E.S. (1977). The valuation of American put options. Journal of Finance, 32(2), 449-462. https://doi.org/10.2307/2326779.

Broadie, M., \& Detemple, J. (1996). American option valuation: new bounds, approximations, and a comparison of existing methods. The Review of Financial Studies, 9(4), 1211-1250. https://doi.org/ 10.1093/rfs/9.4.1211.

Bunch, D.S., \& Johnson, H. (1992). A simple and numerically efficient valuation method for American puts using a modified Geske-Johnson approach. Journal of Finance, 47(2), 809-816. https://doi.org/10.1111/j.1540-6261.1992.tb04412.x.

Byun, S. J., \& Kim, I. J. (1994). Optimal exercise boundary in a binomial option pricing model. Journal of Financial Engineering, 3, 137-158. 
Chen, T., \& Joshi, M. (2012). Truncation and acceleration of the Tian tree for the pricing of American put options. Quantitative Finance, 12(11), 1695-1708. https://doi.org/10.1080/14697688.2011. 617776.

Cox, J. C., Ross, S.A., \& Rubinstein, M. (1979). Option pricing: a simplified approach. Journal of Financial Economics, 7(3), 229-263. https://doi.org/10.1016/0304-405X(79)90015-1.

Curran, M. (1995). Accelerating American option pricing in lattices. Journal of Derivatives, 3(2), 8-18. https://doi.org/10.3905/jod.1995.407936.

Dempster, M. A. H., \& Hutton, J. P. (1999). Pricing American stock options by linear programming. Mathematical Finance, 9(3), 229-254. https://doi.org/10.1111/1467-9965.00069.

Fabozzi, F.J., Paletta, T., Stanescu, S., \& Tunaru, R. (2016). An improved method for pricing and hedging long dated American options. European Journal of Operational Research, 254(2), 656-666. https://doi.org/10.1016/j.ejor.2016.04.002.

Figlewski, S., \& Gao, B. (1999). The adaptive mesh model: a new approach to efficient option pricing. Journal of Financial Economics, 53(3), 313-351. https://doi.org/10.1016/S0304405X(99)00024-0.

Gaudenzi, M., \& Pressacco, F. (2003). An efficient binomial method for pricing American options. Decisions in Economics and Finance, 26(1), 1-17. https://doi.org/10.1007/s102030300000.

Geske, R., \& Johnson, H.E. (1984). The American put option valued analytically. Journal of Finance, 39(5). 1511-1524. https://doi.org/10.1111/j.1540-6261.1984.tb04921.x.

Guo, S., \& Liu, Q. (2019). A simple accurate binomial tree for pricing options on stocks with known dollar dividends. Journal of Derivatives, 26(4), 54-70. https://doi.org/10.3905/jod.2019.26.4.054.

Haug, E.G. (1997). The Complete Guide to Option Pricing Formulas. (2nd ed.). New York: McGrawHill, (Chapter 7).

Huang, J. Z., Subrahmanyam, M.G., \& Yu, G.G. (1996). Pricing and hedging American options: a recursive integration method. Review of Financial Studies, 9(1), 277-300. https://doi.org/10.1093/ rfs/9.1.277.

Joshi, M. S. (2009). The convergence of binomial trees for pricing the American put. Journal of Risk, 11(4), 87-108. https://doi.org/10.21314/JOR.2009.197.

Ju, N., \& Zhong, R. (1999). An approximate formula for pricing American options. Journal of Derivatives, 7(2), 31-40. https://doi.org/10.3905/jod.1999.319140.

Kim, I. J., Jang, B. G., \& Kim, K. T. (2013). A simple iterative method for the valuation of American options. Quantitative Finance, 13(6), 885-895. https://doi.org/10.1080/14697688.2012.696780. 
Leisen, D. P., \& Reimer, M. (1996). Binomial models for option valuation-examining and improving convergence. Applied Mathematical Finance, 3(4), 319-346. https://doi.org/10.1080/ 13504869600000015.

Li, M. (2010). Analytical approximations for the critical stock prices of American options: a performance comparison. Review of Derivatives Research, 13(1), 75-99. https://doi.org/10.1007/ s11147-009-9044-3.

McKean, H. P. (1965). Appendix: A free boundary problem for the heat equation arising from a problem in mathematical economics. Industrial Management Review, 6, 32-39.

Medvedev, A., \& Scaillet, O. (2010). Pricing American options under stochastic volatility and stochastic interest rates. Journal of Financial Economics, 98(1), 145-159. https://doi.org/10.1016/j.jfineco.2010.03.017.

Merton, R. C. (1973). An intertemporal capital asset pricing model. Journal of the Econometric Society, 41, 867-887. https://doi.org/10.2307/1913811.

Nielsen, B. F., Skavhaug, O., \& Tveito, A. (2002). Penalty and front-fixing methods for the numerical solution of American option problems. Journal of Computational Finance, 5(4), 69-97. https://doi.org/10.21314/JCF.2002.084.

Pressacco, F., Gaudenzi, M., Zanette, A., \& Ziani, L. (2008). New insights on testing the efficiency of methods of pricing and hedging American options. European Journal of Operational Research, 185(1), 235-254. https://doi.org/10.1016/j.ejor.2006.12.051

Shang, Q., \& Byrne, B. (2019). American option pricing: an accelerated lattice model with intelligent lattice search. Journal of Derivatives, 27(1), 92-108. https://doi.org/10.3905/jod.2019.1.080.

Staunton, M. (2005). Efficient estimates for valuing American options. The Best of Wilmott 2. New Jersey: John Wiley \& Sons, (Chapter 8).

Tian, Y. (1993). A modified lattice approach to option pricing. Journal of Futures Markets, 13(5), 563577. https://doi.org/10.1002/fut.3990130509.

Wu, L. X., \& Kwok, Y. K. (1997). A front-fixing finite difference method for the valuation of American options. Journal of Financial Engineering, 6(2), 83-97.

Zhu, S.P. (2006). An exact and explicit solution for the valuation of American put options. Quantitative Finance, 6(3), 229-242. https://doi.org/10.1080/14697680600699811. 